\title{
The intrinsic shape of bulges in the CALIFA survey
}

\author{
L. Costantin ${ }^{1}$, J. Méndez-Abreu ${ }^{2,3}$, E. M. Corsini ${ }^{1,4}$, M. C. Eliche-Moral ${ }^{2}$, T. Tapia ${ }^{5}$, L. Morelli ${ }^{1,4}$, \\ E. Dalla Bontà ${ }^{1,4}$, and A. Pizzella ${ }^{1,4}$
}

\author{
1 Dipartimento di Fisica e Astronomia “G. Galilei”, Università di Padova, vicolo dell'Osservatorio 3, 35122 Padova, Italy \\ e-mail: luca.costantin@studenti.unipd.it \\ 2 Instituto de Astrofísica de Canarias, Calle vía Láctea s/n, 38200 La Laguna, Tenerife, Spain \\ 3 Departamento de Astrofísica, Universidad de La Laguna, Calle Astrofísico Francisco Sánchez s/n, 38205 La Laguna, \\ Tenerife, Spain \\ ${ }^{4}$ INAF-Osservatorio Astronomico di Padova, vicolo dell'Osservatorio 5, 35122 Padova, Italy \\ 5 Instituto de Astronomía, Universidad Nacional Autónoma de México, Apdo. 106, Ensenada BC 22800, Mexico
}

Received 24 August 2017 / Accepted 13 October 2017

\begin{abstract}
Context. The intrinsic shape of galactic bulges in nearby galaxies provides crucial information to separate bulge types. Aims. We aim to derive accurate constraints to the intrinsic shape of bulges to provide new clues on their formation mechanisms and set new limitations for future simulations.

Methods. We retrieved the intrinsic shape of a sample of CALIFA bulges using a statistical approach. Taking advantage of GalMer numerical simulations of binary mergers we estimated the reliability of the procedure. Analyzing the $i$-band mock images of resulting lenticular remnants, we studied the intrinsic shape of their bulges at different galaxy inclinations. Finally, we introduced a new $(B / A$, $C / A$ ) diagram to analyze possible correlations between the intrinsic shape and the properties of bulges.

Results. We tested the method on simulated lenticular remnants, finding that for galaxies with inclinations of $25^{\circ} \leq \theta \leq 65^{\circ}$ we can safely derive the intrinsic shape of their bulges. We found that our CALIFA bulges tend to be nearly oblate systems $(66 \%)$, with a smaller fraction of prolate spheroids (19\%), and triaxial ellipsoids (15\%). The majority of triaxial bulges are in barred galaxies (75\%). Moreover, we found that bulges with low Sérsic indices or in galaxies with low bulge-to-total luminosity ratios form a heterogeneous class of objects; additionally, bulges in late-type galaxies or in less massive galaxies have no preference for being oblate, prolate, or triaxial. On the contrary, bulges with high Sérsic index, in early-type galaxies, or in more massive galaxies are mostly oblate systems. Conclusions. We concluded that various evolutionary pathways may coexist in galaxies, with merging events and dissipative collapse being the main mechanisms driving the formation of the most massive oblate bulges and bar evolution reshaping the less massive triaxial bulges.
\end{abstract}

Key words. galaxies: bulges - galaxies: evolution - galaxies: formation - galaxies: fundamental parameters - galaxies: photometry galaxies: structure

\section{Introduction}

In observational extragalactic astrophysics, our measurements of the light distribution of galaxies are confined to the twodimensional framework of the sky plane. Although observations can only access the projected rather than intrinsic luminosity density of galaxies, we can disentangle their different luminous components, including bulges. Constraining the threedimensional light distribution of the galaxy components, and therefore their intrinsic shape, is a crucial piece of information in our understanding of how galaxies form and evolve.

Several studies have addressed the intrinsic shape of the elliptical galaxies (Sandage et al. 1970; Tremblay \& Merritt 1996; Rodríguez \& Padilla 2013). Although many of ellipticals were initially thought to be oblate or prolate spheroids, some photometric (i.e., the twisting of the isophotes; Carter 1978; Bertola \& Galletta 1979) and kinematic properties (i.e., the low rotation of stars or the kinematic misalignment; Bertola \& Capaccioli 1975; Illingworth 1977; Krajnović et al. 2011) promptly supported the idea that some of them could be triaxial ellipsoids. In general, faint ellipticals are more flattened with a tendency to be oblate spheroids, whereas bright ellipticals are rounder and more frequently triaxial ellipsoids
(Weijmans et al. 2014). It should be noticed that most of the works about the intrinsic shape of ellipticals deal with the distribution function of the intrinsic axial ratios of the whole population of ellipticals through statistical analyses of their apparent flattenings (see Méndez-Abreu 2016, for a review). As a matter of fact, it is not possible to recover the intrinsic shape of an individual elliptical galaxy by only studying its light distribution (Statler et al. 2001). Indeed, deprojecting the apparent shape of an elliptical into its intrinsic shape represents a typical ill-posed problem, caused by the lack of observational constraints on the three Euler angles $\theta, \phi, \psi)$ that provide the transformation. Further details about the galaxy structure, like the presence of dust lanes, gaseous disks or embedded stellar disks (e.g., NGC 5077, Bertola et al. 1991a) or the knowledge of the stellar velocity field (e.g., NGC 4365, van den Bosch et al. 2008) are needed to overcome this problem.

On the contrary, in disk galaxies it is possible to derive the intrinsic shape of individual bulges because of the presence of the disk component, whose observed ellipticity provides a proxy for the bulge inclination, under the assumptions that both the bulge and disk share the same equatorial plane of symmetry and that disks are highly flattened oblate spheroids (but see also Ryden 2004, 2006). 
The bulge is photometrically defined as the structural component responsible for the light excess measured in the galaxy central regions, above the inward extrapolation of the exponential surface-brightness profile of the disk (Andredakis et al 1995; Balcells et al. 2007). The main concern for bulges is separating their light contribution from that of the other galaxy components. This is usually done by means of the photometric decomposition of the galaxy surface brightness into the contribution of the bulge and disk, and possibly of a lens, a bar, inner or outer rings, nuclear unresolved components, and the spiral arms (Peng et al. 2002; Laurikainen et al. 2005; Gadotti 2009; Benítez et al. 2013; Erwin 2015).

Nowadays, it is widely accepted that bulges are not simple axisymmetric structures in the center of galaxies (see Méndez-Abreu 2016, for a review). The misalignment between the bulge and disk isophotes observed in many spirals (e.g., M31, Lindblad 1956; Williams \& Schwarzschild 1979) resembles the isophotal twist of ellipticals and it is similarly interpreted as the signature of bulge triaxiality. The first quantitative estimate of the triaxiality of bulges was carried out by Bertola et al. (1991b) by studying the misalignment between the major axes of the bulge and disk in a sample of 32 early-type disk galaxies. They found that the mean intrinsic axial ratio of bulges in the disk plane is $\langle B \mid A\rangle=0.86$, while their mean intrinsic flattening in the plane perpendicular to the disk plane is $\langle C \mid A\rangle=0.65$, where $A, B$, and $C$ are the lengths of the semi-axes of the bulge ellipsoid. This result was later confirmed by Fathi \& Peletier (2003), who analyzed the deprojected axial ratio of the galaxy isophotes within the bulge radius in a sample of 70 disk galaxies, ranging from lenticulars to late-type spirals. They found $\langle B / A\rangle=0.79$ and $\langle B \mid A\rangle=0.71$ for the bulges in earlier and later morphological types, respectively. By means of a two-dimensional photometric decomposition, Méndez-Abreu et al. (2008) measured the structural parameters of both bulges and disks in a sample of 148 early-to-intermediate spirals, increasing the statistics of Bertola et al. (1991b) by an order of magnitude. They found that about $80 \%$ of the sample bulges are triaxial ellipsoids with $\langle B / A\rangle=0.85$. More recently, Méndez-Abreu et al. (2010) introduced a novel statistical method to constrain the intrinsic shape of individual bulges. The knowledge of the geometric properties (i.e., the apparent ellipticity and major-axis position angle) of the bulge and disk makes it possible to simultaneously compute the probability distribution function of the intrinsic axial ratios $B / A$ and $C / A$ for every single bulge. They revisited the galaxies of the sample of Méndez-Abreu et al. (2008) and concluded that $65 \%$ of them host oblate triaxial bulges while the remaining ones have prolate triaxial bulges.

Nevertheless, further efforts are required to better characterize the intrinsic shape of bulges and, in particular, a higher number statistics is needed to investigate the correlations between the bulge shape and galaxy properties. Knowing the intrinsic shape of bulges completes our understanding of the potential well and orbital distribution of the stars in the inner regions of galaxies. This will also help us to explain the origin of the different populations of classical and disk-like bulges as well as to address the assembly processes of their host galaxies. (e.g., Athanassoula 2005; Brooks \& Christensen 2016). The general agreement on bulge formation is that rapid dissipative collapses (Eggen et al. 1962; Sandage 1990) or the violent relaxation by galactic major merger events (Toomre 1977; Kauffmann 1996) form classical bulges, resembling oblate spheroids with intermediate or low flattening and with a certain degree of triaxiality. Numerical simulations have also demonstrated the relevance of minor mergers in the build up of a classical bulge
(Aguerri et al. 2001; Eliche-Moral et al. 2006). In the context of galaxy formation, cosmological simulations highlighted that the actual population of galaxies can be represented only by the proper combination of major and minor, gas-rich and gas-poor mergers (Oser et al. 2012; Naab et al. 2014). Moreover, the recurring coalescence of long-lived giant star-forming clumps at high redshift was also proposed as a mechanism for the formation of classical bulges (Dekel et al. 2009; Ceverino et al. 2015). On the contrary, secular processes linked to the evolution of galactic substructures (i.e., bars, lenses, ovals, etc.) reshape the center of galaxies into either boxy/peanut components (Erwin \& Debattista 2013; Laurikainen et al. 2014) or more flattened disk-like bulges (Kormendy \& Kennicutt 2004; Kormendy 2016). In this scenario, disk-like bulges are expected to be axisymmetric systems, whereas boxy/peanut structures show some degree of triaxiality (Athanassoula \& Beaton 2006), being the vertically-thick inner parts of bars resulting from buckling or resonant effects (Combes \& Sanders 1981; Lütticke et al. 2000). Another possible mechanism invoked for disk-like bulge growth is the fast disruption of short-lived giant clumps at high redshift, if no relaxation processes affect the central region of the galaxy (Hopkins et al. 2012; Bournaud 2016).

Currently, the observational separation between classical and disk-like bulges is usually done by analyzing their observed photometric, kinematic, or stellar population properties (Morelli et al. 2008; Coelho \& Gadotti 2011). However, the demarcation lines are often blurred making difficult to understand the actual frequency of different bulge types (Costantin et al. 2017). Furthermore, bulges can suffer from different processes during their lifetime with some of them giving rise to similar observational properties. As a consequence, different kind of bulges can coexist in the same galaxy (Athanassoula 2005; Méndez-Abreu et al. 2014; Erwin et al. 2015). Nonetheless, the measurements of the intrinsic shape of bulges might provide a fundamental additional constraint to separate bulge types, as well as limitations for future numerical simulations willing to reproduce realistic galaxies.

In this paper, we analyze the intrinsic shape of the bulges of some of the disk galaxies observed in the Calar Alto Legacy Integral Field Area survey Data Release 3 (CALIFA DR3; Sánchez et al. 2016). We aim at investigating the possible links between the intrinsic shape of bulges and their observed photometric properties. Here, we improve the previous results by Méndez-Abreu et al. $(2008,2010)$ by testing the reliability of their statistical method and setting limits on the galaxy inclination to its successful application with the help of mock images of a set of simulated remnant galaxies. The paper is organized as follows. We present the galaxy sample in Sect. 2. We summarize the statistical method for retrieving the intrinsic shape of bulges in Sect. 3. We make use of mock images of simulated remnant galaxies seen at different inclinations to understand the limits of our analysis in Sect. 4. We derive the intrinsic shape of the bulges of the sample galaxies in Sect. 5. We discuss the possible implications of our results for galaxy formation in Sect. 6. We summarize our findings in Sect. 7. We adopt $H_{0}=70 \mathrm{~km} \mathrm{~s}^{-1} \mathrm{Mpc}^{-1}$, $\Omega_{\mathrm{M}}=0.3$, and $\Omega_{\Lambda}=0.7$ as cosmological parameters throughout this work.

\section{Sample selection}

We selected our galaxies sample from the final sample of galaxies included in the CALIFA DR3, which was drawn from the Sloan Digital Sky Survey (SDSS) DR7 (Abazajian et al. 2009) and comprises 667 nearby galaxies $(0.005<z<0.03)$ with an 


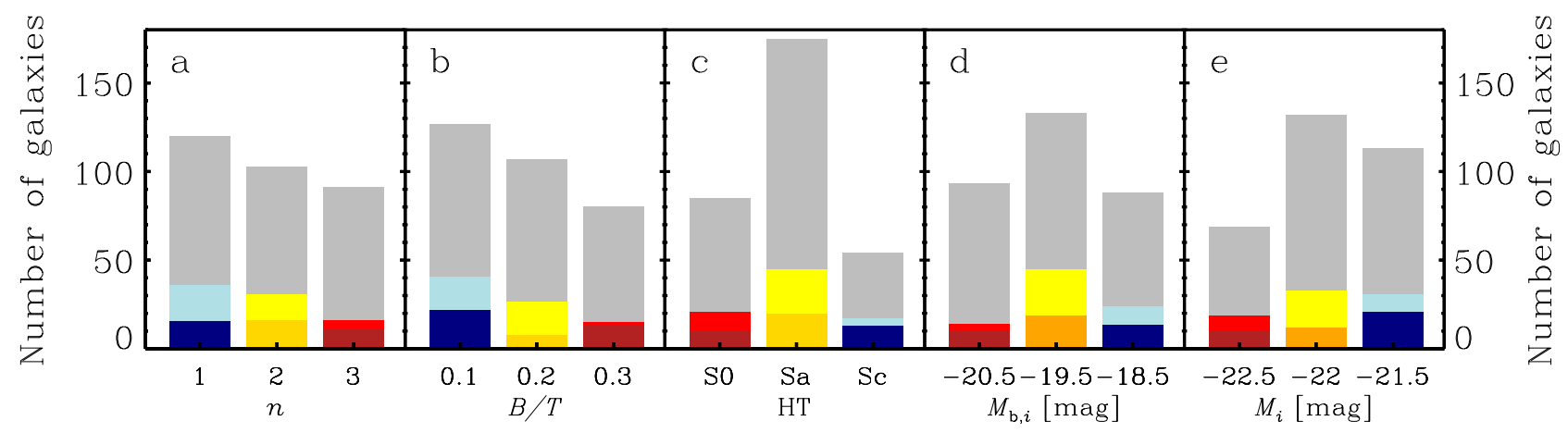

Fig. 1. Distribution of the Sérsic index of the bulge (panel a), bulge-to-total luminosity ratio (panel b), Hubble type (panel c; Sa bin comprises $\mathrm{Sa}-\mathrm{Sab}$-Sb-Sbc galaxies, while Sc bin comprises Sc-Scd-Sd-Sdm galaxies), $i$-band absolute magnitude of the bulge (panel $d$ ), and $i$-band absolute magnitude of the galaxy (panel e) for our final sample of 43 unbarred (dark color histograms) and 40 barred galaxies (light color histograms). The gray histograms show the distribution of the remaining 231 galaxies of the sample of 314 disk galaxies selected from CALIFA DR3. The galaxy properties are taken from Méndez-Abreu et al. (2017).

angular isophotal diameter between 45 and 79.2 arcsec at a surface brightness level of $25 \mathrm{mag} \operatorname{arcsec}^{-2}$ in the $r$ band.

First, we focused onto the 314 disk galaxies of CALIFA DR3, not interacting or merging, with a photometric decomposition obtained by Méndez-Abreu et al. (2017). These galaxies were fit with either bulge and disk only (177 galaxies), or with a bar in addition to bulge and disk (137 galaxies) using the GAlaxy Surface Photometry 2 Dimensional Decomposition code (GASP2D; Méndez-Abreu et al. 2008, 2014).

Then, we took into account only the galaxies with good imaging, that is the absence of either strong fluctuations of the local sky background around the galaxy or other bright component affecting the photometric decomposition (e.g., a lens, inner and/or outer rings, and spiral arms), and all the structural parameters of the bulge, disk, and bar left free to vary during the fitting process (i.e., the galaxies flagged as 1 ,a in Table 1 of Méndez-Abreu et al. 2017). This allowed us to obtain a subsample of 118 robustly fit galaxies (67 unbarred and 51 barred galaxies), with no bias on the measured structural parameters that could hamper our analysis of the bulge intrinsic shape.

Finally, we set a limit onto the galaxy inclination $\left(25^{\circ}<\theta<\right.$ $65^{\circ}$ ) to exclude both the low-inclined galaxies, for which it is not possible to constrain the bulge shape along the direction perpendicular to the disk plane, and the highly-inclined ones, for which the results of the GASP2D photometric decomposition are not reliable (Méndez-Abreu et al. 2017) and the bulge shape on the disk plane is unconstrained (see Sect. 4.3 for a discussion). This selection criterion reduced the galaxy sample to 83 objects (43 unbarred and 40 barred galaxies), as shown in Tables C.1 and C.2.

We considered only galaxies with $i$-band images to better resolve the bulge component minimizing the dust effects with respect to the other SDSS passbands. The choice of $i$ band assured a sufficient spatial resolution $(F W H M=1.1 \pm 0.2 \operatorname{arcsec})$ and depth (out to $\mu_{i} \simeq 26 \mathrm{mag} \operatorname{arcsec}^{-2}$ ), as retrieved from Méndez-Abreu et al. (2017). Their basic properties (i.e., Sérsic index of the bulge $n$, bulge-to-total luminosity ratio $B / T$, Hubble type HT, $i$-band absolute magnitude of the bulge $M_{\mathrm{b}, i}$, and $i$-band absolute magnitude of the galaxy $M_{i}$ from Méndez-Abreu et al. 2017) are shown in Fig. 1 and compared with those of the selected sample of 314 disk galaxies from CALIFA DR3. This final sample is not complete in volume. However, we thought that the selection in diameter of the CALIFA sample should not introduce any major bias in our results, since the distribution of bulge observed properties is well sampled, as shown in Fig. 1.

\section{Bulge intrinsic shape}

The full description of the statistical method adopted to derive the intrinsic shape of the bulges of our galaxy sample is given in Méndez-Abreu et al. (2010). Here, for the sake of clarity, we have summarized the main hypotheses and gave the most relevant equations that link the intrinsic axial ratios of the bulge to the observed properties of the galaxy. In particular, we rewrote the description of the probability function $P(B / A)$ (Eq. (34) in Méndez-Abreu et al. 2010) and we revised the equation linking the axial ratios $B / A$ and $C / A$ (Eq. (59) in Méndez-Abreu et al. 2010) and implemented it in the galaXYZ code written in IDL $^{1}$.

\subsection{Bulge and disk geometry}

In order to characterize the intrinsic shape of a bulge, we assumed it to be a triaxial ellipsoid with the same equatorial plane as the disk, which we supposed to be infinitesimally thin. Moreover, the bulge and disk share the same center, which coincides with the galaxy center (Fig. 2).

Let $(x, y, z)$ be the Cartesian coordinates in the reference system of the galaxy. The origin of the system is in the galaxy center, the $x$-axis and $y$-axis correspond to the bulge principal axes in the equatorial plane, while the $z$-axis corresponds to the common polar axis of both the bulge and disk. The equation of the bulge in its own reference system is given by

$$
\frac{x^{2}}{A^{2}}+\frac{y^{2}}{B^{2}}+\frac{z^{2}}{C^{2}}=1
$$

where $A, B$, and $C$ are the lengths of the bulge intrinsic semi-axes.

Let $\left(x^{\prime}, y^{\prime}, z^{\prime}\right)$ be the Cartesian coordinates in the reference system of the observer. The origin of the system is in the galaxy center, the polar $z^{\prime}$-axis is along the line of sight (LOS) and points toward the galaxy, while $\left(x^{\prime}, y^{\prime}\right)$ confines the sky plane.

The intersection between the bulge equatorial plane $(x, y)$ and the sky plane $\left(x^{\prime}, y^{\prime}\right)$ is the so-called line of nodes (LON). The angle $\theta$ between the polar $z$-axis and the polar $z^{\prime}$-axis defines the bulge inclination. Let $\phi$ be the angle between the $x$-axis and the LON in the bulge equatorial plane and let $\psi$ be the angle between the $x^{\prime}$-axis and the LON in the sky plane. The three Euler angles $(\theta, \phi, \psi)$ allow for the transformation from the reference

1 Interactive Data Language is distributed by ITT Visual Information Solutions. It is available from http://www.ittvis. com 


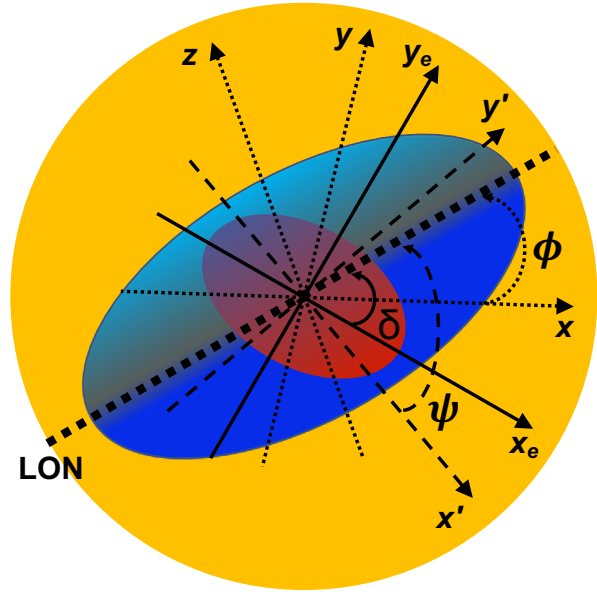

Fig. 2. Schematic three-dimensional view of a galaxy with a triaxial bulge and a infinitesimally thin disk. The bulge is shown as seen by the observer along the LOS. The bulge, disk plane, and sky plane are plotted in red, blue, and orange, respectively. The reference systems of the galaxy $(x, y, z)$ and observer $\left(x^{\prime}, y^{\prime}, z^{\prime}\right)$ as well as the LON are plotted with thin dotted lines, thin dashed lines, and a thick dashed line, respectively. The axes of symmetry $\left(x_{\mathrm{e}}, y_{\mathrm{e}}\right)$ of the bulge ellipse in the sky plane are represented with thin solid lines.

system of the sky to that of the galaxy. If the $x^{\prime}$-axis coincides with the LON, consequently it is $\psi=0$.

The projection onto the sky plane of the triaxial ellipsoid given in Eq. (1) is an ellipse which corresponds on the galaxy image to the photometric bulge. It is given by

$\frac{x_{\mathrm{e}}^{2}}{a^{2}}+\frac{y_{\mathrm{e}}^{2}}{b^{2}}=1$,

where $x_{\mathrm{e}}$ and $y_{\mathrm{e}}$ are taken along the symmetry axes of the bulge ellipse, while $a$ and $b$ are lengths of the ellipse semi-major and semi-minor axis, respectively. The twist angle $\delta$ between the $x_{\mathrm{e}^{-}}$ axis and the LON indicates the bulge orientation. We always consider $0<\delta<90^{\circ}$ such that $a$ can be either the major or the minor semi-axis.

The twist angle $\delta$ and apparent axial ratio of the bulge $\left(q_{\mathrm{b}}=b / a\right)$ depend only, and unambiguously, on the direction of the $\operatorname{LOS}$ (i.e., on $\theta, \phi$, and $\psi$ ) and on the intrinsic shape of the bulge (i.e., on $A, B$, and $C$ ). Thus, by means of the Euler angles it is possible to impose further constraints to the equations that relate the intrinsic parameters of the bulge in the reference system of the galaxy to the observed properties of the galaxy in the reference system of the observer (Simonneau et al. 1998; Méndez-Abreu et al. 2008). Introducing physical constraints on the accessible viewing angles (e.g., imposing that $A, B$, and $C$ must be definite positive), it is possible to statistically derive the intrinsic axial ratios of the bulge from its observed properties. Unfortunately, the problem is not analytically solved because of the unknown spatial position of the bulge (i.e., the angle $\phi$ ), which constitutes the basis of the statistical analysis (see Méndez-Abreu et al. 2010, for all the details).

\subsection{Statistical analysis}

The theoretical framework based on the statistical analysis of the $\phi$ angle allows us to retrieve the intrinsic shape of individual bulges in disk galaxies from the bulge apparent shape (i.e., the bulge ellipticity $\epsilon_{\mathrm{b}}=1-q_{\mathrm{b}}$ ), the disk apparent shape (i.e., the disk ellipticity $\epsilon_{\mathrm{d}}=1-q_{\mathrm{d}}$, where $q_{\mathrm{d}}$ is the apparent axial ratio of the disk), and the bulge twist angle (i.e., the difference between the position angles of the bulge and disk $\delta=\mathrm{PA}_{\mathrm{d}}-\mathrm{PA}_{\mathrm{b}}$; see Méndez-Abreu et al. 2010 for all details).

The apparent axial ratio of a circular and infinitesimally thin disk is a measure of the bulge inclination

$\theta=\arccos q_{\mathrm{d}}$,

when both the bulge and disk share the same equatorial plane. However, disks are not infinitely thin structures (Sandage et al. 1970; Ryden 2004). To account for this, we computed the inclination of our galaxies accounting for the distribution function of the intrinsic axial ratio of the disks $q_{0, \mathrm{~d}}$. We adopted a normal distribution function with mean intrinsic axial ratio $\left\langle q_{0, \mathrm{~d}}\right\rangle=0.267$ and standard deviation $\sigma_{q_{0, \mathrm{~d}}}=0.102$ following Rodríguez \& Padilla (2013). Thus, the statistical value of the galaxy inclination is

$\theta=\arccos \sqrt{\frac{q_{\mathrm{d}}^{2}-q_{0, \mathrm{~d}}^{2}}{1-q_{0, \mathrm{~d}}^{2}}}$,

where the $q_{0, \mathrm{~d}}$ value is randomly drawn from the previous normal distribution.

We took into account the uncertainties in $\epsilon_{\mathrm{b}}, \epsilon_{\mathrm{d}}$, and $\delta$ derived from the error analysis of the photometric decomposition (Méndez-Abreu et al. 2017). We randomly generated 1000 geometric configurations by adopting for each parameter a Gaussian distribution centered on its measured value and with a standard deviation equal to its uncertainty. A similar analysis was introduced by Corsini et al. (2012) to recover the intrinsic shape of the polar bulge of NGC 4698. For each geometric configuration, we calculated 5000 values of $B / A$ using Monte Carlo simulations and according to its probability function

$P\left(\frac{B}{A}\right)=\frac{2 \frac{B}{A} \sin \phi_{B}}{\left(\phi_{C}-\phi_{B}\right)\left(1-\frac{B^{2}}{A^{2}}\right) \sqrt{\left(1-\frac{B^{2}}{A^{2}}\right)^{2}-\sin ^{2} \phi_{B}\left(1+\frac{B^{2}}{A^{2}}\right)^{2}}}$,

where $\phi_{B}$ and $\phi_{C}$ are the angles where the length of the intrinsic semi-axis $B$ and $C$ are zero, respectively. Since $B / A$ and $C / A$ are both functions of the same variable $\phi$, their probabilities $P(B / A)$ and $P(C / A)$ are equivalent; thus, after sampling the value of $B / A$ using Eq. (5), we calculated the value of $C / A$ using

$$
\begin{aligned}
\frac{2 \sin \left(2 \phi_{C}\right)}{F_{\theta}} \frac{C^{2}}{A^{2}}= & \sin \left(2 \phi_{C}-\phi_{B}\right) \sqrt{\left(1-\frac{B^{2}}{A^{2}}\right)^{2}-\sin ^{2} \phi_{B}\left(1+\frac{B^{2}}{A^{2}}\right)^{2}} \\
& -\sin \phi_{B} \cos \left(2 \phi_{C}-\phi_{B}\right)\left(1+\frac{B^{2}}{A^{2}}\right)
\end{aligned}
$$

where $\phi_{B}, \phi_{C}$, and $F_{\theta}$ are functions of the observed quantities $a$, $b, \delta$, and $\theta$ (see Méndez-Abreu et al. 2010, for a full description of the different variables).

\section{Bulge intrinsic shape of the simulated lenticular remnants}

\subsection{Simulated lenticular remnants from numerical experiments of binary mergers}

In order to test our statistical method for recovering the intrinsic shape of bulges and to understand its limitations, we 
Table 1. Intrinsic shape of the bulges of the simulated lenticular remnants seen at different inclinations.

\begin{tabular}{lcccccccc}
\hline \hline \multicolumn{1}{c}{ Galaxy } & \multicolumn{9}{c}{$B / A$} & & & \multicolumn{3}{c}{$C / A$} \\
\multicolumn{1}{c}{$(1)$} & $\left(0^{\circ}\right)$ & $\left(180^{\circ}\right)$ & $\begin{array}{c}\left(30^{\circ}\right) \\
(4)\end{array}$ & $\left(45^{\circ}\right)$ & $\left(60^{\circ}\right)$ & $\left(30^{\circ}\right)$ & $\left(45^{\circ}\right)$ & $\left(60^{\circ}\right)$ \\
$(6)$ & $(7)$ & $(8)$ & $(9)$ \\
\hline gE0gSbo5 & 1.00 & 1.00 & 1.00 & 1.00 & 1.00 & 0.99 & 0.59 & 0.59 \\
gE0gSdo5 & 0.79 & 0.79 & 0.91 & 0.89 & 0.86 & 0.46 & 0.26 & 0.26 \\
gS0dE0o98 & 0.46 & 0.49 & 0.49 & 0.64 & 0.56 & 0.51 & 0.21 & 0.36 \\
gS0dE0o99 & 0.46 & 0.46 & 0.54 & 0.59 & 0.69 & 0.64 & 0.64 & 0.64 \\
gS0dE0o100 & 0.46 & 0.46 & 0.49 & 0.54 & 0.56 & 0.59 & 0.14 & 0.39 \\
gS0dS0099 & 0.59 & 0.59 & 0.56 & 0.61 & 0.76 & 0.64 & 0.41 & 0.21 \\
gS0dSao103 & 0.51 & 0.51 & 0.51 & 0.56 & 0.56 & 0.96 & 0.21 & 0.34 \\
gS0dSbo106 & 0.44 & 0.44 & 0.41 & 0.46 & 0.61 & 0.69 & 0.39 & 0.29 \\
gS0dSdo100 & 0.51 & 0.51 & 0.49 & 0.51 & 0.74 & 0.54 & 0.41 & 0.24 \\
gSbgSb09 & 0.71 & 0.71 & 0.76 & 0.76 & 0.76 & 1.06 & 0.74 & 0.54 \\
gSbgSdo5 & 0.81 & 0.84 & 0.84 & 0.81 & 0.84 & 0.36 & 0.26 & 0.21 \\
\hline
\end{tabular}

Notes. (1) Identifier in the GalMer database of the merger experiment resulting in a lenticular remnant, which we adopted as the name of the simulated galaxy. (2)-(6) Intrinsic axial ratio $B / A$ of the bulge obtained from the mock images of the simulated lenticular remnants seen at an inclination $\theta=0^{\circ}, 180^{\circ}, 30^{\circ}, 45^{\circ}$, and $60^{\circ}$, respectively. (7)-(9) Intrinsic axial ratio $C / A$ of the bulge obtained from the mock images of the simulated lenticular remnants seen at an inclination $\theta=30^{\circ}, 45^{\circ}$, and $60^{\circ}$, respectively.

measured the intrinsic axial ratios of the bulge against different galaxy inclinations. To this aim, we used a subset of 11 numerical simulations from the GalMer database ${ }^{2}$ (Chilingarian et al. 2010). We used remnant galaxies of a variety of merger experiments between pairs of galaxies with different mass, morphology, gas content, and orbital parameters. These remnant galaxies strongly resemble lenticular galaxies, according to their morphological, photometric, and kinematic properties (Borlaff et al. 2014; Querejeta et al. 2015a,b; Tapia et al. 2017; Eliche-Moral et al., in prep.).

We chose to analyze simulated lenticular remnants resulting from binary mergers instead of $N$-body realizations of analytical expressions, as those adopted for building the progenitor galaxies of the remnants, because we required a certain degree of bulge triaxiality. Such a triaxiality is a common feature of merger remnants, although their progenitors could be axisymmetric by construction (Cox et al. 2006; Tapia et al. 2014). All the simulated lenticular remnants of the analyzed merger experiments are either unbarred galaxies if resulting from a major merger, or weakly barred galaxies if resulting from a minor merger (Eliche-Moral et al., in prep.). We discarded the merger experiments producing elliptical or $\mathrm{E} / \mathrm{S} 0$ remnants, in order to have simulated galaxies with a well-defined bulge embedded into a large disk, similarly to the observed CALIFA galaxies.

The progenitor galaxies were modeled with a spherical nonrotating dark-matter halo, which contains a stellar and/or a gaseous disk and/or a central non-rotating bulge, depending on their morphological type. The primary galaxy consisted of a giant galaxy (hereafter gE0 for a giant-like elliptical, gS0 for a giant-like lenticular, gSa for a giant-like Sa spiral, gSb for a giant-like Sb spiral, and gSd for a giant-like Sd spiral) interacting either with another giant galaxy of similar mass or with a dwarf galaxy (hereafter dE0, dS0, dSa, dSb, and dSd), whose total mass is ten times smaller than that of the giant galaxy. Several simulations were performed varying the initial orbital energy, pericenter distance and inclination with respect to the orbital plane of the interacting galaxies. Indeed, for each interacting pair the disk (when present) of one of the two galaxies is kept in the orbital plane, while the companion disk can have a

\footnotetext{
2 The GalMer database is a public library of hydrodynamics $N$-body simulations of galaxy mergers with intermediate resolution available at http://www.project-horizon.fr/
}

different inclination. Direct and retrograde orbits were also taken into account, where direct or retrograde spin-orbit coupling refer to progenitors having either parallel or antiparallel spins, respectively. The merger experiments have a total of 240000 and 528000 particles for the major and minor merger events, respectively. The particles have a mass $M=3.5-20.0 \times 10^{5} M_{\odot}$ each. The merger experiments have a duration of 3-3.5 Gyr and were evolved using a Tree-SPH code (Semelin \& Combes 2002), adopting the same softening length for all particle types $\epsilon=280 \mathrm{pc}$ for the giant-giant galaxy mergers and $\epsilon=200 \mathrm{pc}$ for the giant-dwarf galaxy mergers. The effects of gas and star formation (SF; such as the stellar mass loss, metallicity enrichment of the interstellar medium, and energy injection due to supernova explosions) were considered using the method described in Mihos \& Hernquist (1994). The stellar mass of the lenticular remnants is in the range $M=1-3 \times 10^{11} M_{\odot}$ for major mergers and $M=1.2-1.3 \times 10^{11} M_{\odot}$ for minor ones.

The merger experiments we analyzed were chosen to cover the whole range of morphologies, mass ratios, and orbital configurations of the progenitors. They are listed in Table 1 and labeled considering both the morphological type of the progenitors and the unique numerical identifier given to the orbit in the GalMer database. The orbital configuration of each merger experiment is provided in Table 2. For example, the experiment gS0dE0o100 corresponds to the accretion of a dwarf elliptical by a giantlike lenticular. It follows the orbit tagged as 100 in the GalMer database with an inclination of $33^{\circ}$ with respect to the orbital plane, a pericenter distance of $8 \mathrm{kpc}$, and a initial energy of $15 \times 10^{4} \mathrm{~km}^{2} \mathrm{~s}^{-2}$ in a prograde spin-orbit coupling.

\subsection{Photometric decomposition of the simulated lenticular remnants seen at different inclinations}

To perform a fair comparison between the results from our sample of simulated bulges and the final observed sample from CALIFA, we built mock images of the simulated lenticular remnants under the observing setup of the CALIFA galaxies.

Therefore, we mimicked SDSS $i$-band images of the simulated lenticular remnants assuming they are at a distance of $67 \mathrm{Mpc}$, which corresponds to the median distance of the CALIFA DR3 galaxies. We modeled the point spread function (PSF) with a circular Moffat profile (Moffat 1969) with $F W H M=1.2 \operatorname{arcsec}$ and $\beta=5$, which represent typical 
Table 2. Orbital parameters of the merger experiments resulting in the simulated lenticular remnants listed in Table 1.

\begin{tabular}{ccccc}
\hline \hline $\mathrm{ID}_{\text {orb }}$ & $\begin{array}{c}\text { Spin-orbit } \\
{[\mathrm{P} / \mathrm{R}]}\end{array}$ & $\begin{array}{c}i_{2} \\
{\left[{ }^{\circ}\right]}\end{array}$ & $\begin{array}{c}d_{\text {per }} \\
{[\mathrm{kpc}]}\end{array}$ & $\begin{array}{c}E_{0} \\
\left(10^{4} \mathrm{~km}^{2} \mathrm{~s}^{-2}\right] \\
(1)\end{array}$ \\
$(2)$ & $(3)$ & $(4)$ & $(5)$ \\
\hline 5 & $\mathrm{P}$ & 0 & 16 & 0 \\
9 & $\mathrm{P}$ & 0 & 24 & 0 \\
98 & $\mathrm{P}$ & 33 & 8 & 2.5 \\
99 & $\mathrm{P}$ & 33 & 8 & 5 \\
100 & $\mathrm{P}$ & 33 & 8 & 15 \\
103 & $\mathrm{R}$ & 33 & 8 & 0 \\
106 & $\mathrm{R}$ & 33 & 8 & 15 \\
\hline
\end{tabular}

Notes. (1) Identifier in the GalMer database of the orbit used in the merger experiment. (2) Spin-orbit coupling (P: prograde; R: retrograde). (3) Inclination of the secondary progenitor with respect to the orbital plane. (4) Pericenter distance. (5) Initial orbital energy.

values for the SDSS images of the galaxies in CALIFA DR3 (Méndez-Abreu et al. 2017). Moreover, we considered a Poissonian photon noise to yield signal-to-noise ratio, $S / N=1$ at a limiting magnitude of $\mu_{i}=25.7 \mathrm{mag} \operatorname{arcsec}^{-2}$. We chose a pixel scale of 0.396 arcsec pixel ${ }^{-1}$ and, for simplicity, we assumed a gain of $1 e^{-} \mathrm{ADU}^{-1}$ and a readout noise of $1 e^{-} \mathrm{rms}$.

We converted the mass of each particle of the simulated lenticular remnants into light by adopting the $i$-band mass-tolight ratio $(M / L)$ corresponding to the stellar population of the same age and metallicity of the particle. For the old stellar particles, we assumed that they have evolved previous to the merger following a typical star formation history (SFH), according to the morphological type of the progenitors as found in real galaxies (Eliche-Moral et al. 2010). Since the SF is transferred to the hybrid particles at the start of the merger simulation, the $\mathrm{SFH}$ of the old stellar particles is stopped at that moment and they are assumed to evolve passively since then. We have thus adopted a present-day age of $11 \mathrm{Gyr}$ for the old stellar component because it is the average age of the old stellar population in the disks of nearby lenticular galaxies (Sil'chenko et al. 2012; Sil'chenko 2013). The SFHs were estimated using the stellar population synthesis models by Bruzual \& Charlot (2003) with a Chabrier initial mass function (Chabrier 2003), and the evolutionary tracks by Bertelli et al. (1994). Concerning the hybrid particles, the SF in the galaxies that merge is transferred to them during the simulation. So, part of their initial mass (totally gaseous at the start of the simulation) turns into stellar mass during the merger depending on the local gas concentration. The SFH of these particles is specifically computed during the experiment and it is different for each particle (Chilingarian et al. 2010). Although it may be quite complex, most of their SF accumulates into one or two short peaks occurred soon after the first pericenter passage and the full merger, mostly in this last one (see Di Matteo et al. 2007, 2008; Lotz et al. 2008; and Eliche-Moral et al., in prep.). Therefore, we have approximated the complex SFH of each hybrid particle by simple stellar populations (SSPs), assuming the mean age and metallicity that each hybrid particle presents at the end of the simulation, to estimate $M / L$ for each one and convert their newly formed stellar mass into luminosity. For this goal, we have used the same stellar population synthesis models commented before. We transformed the intrinsic physical values of lengths into projected angular values and we corrected the resulting surface brightness by cosmological dimming (see Tapia et al. 2017, for more details).
For each simulated lenticular remnant, we created six mock images corresponding to different inclinations with respect to the direction of the total angular momentum vector of the simulated lenticular remnant (i.e., $\theta=0^{\circ}, 30^{\circ}, 45^{\circ}, 60^{\circ}, 90^{\circ}$, and $180^{\circ}$ ). The face-on views $\theta=0^{\circ}$ and $180^{\circ}$ correspond to the cases where the angular momentum vector points toward to and away from the observer, respectively. This allowed us to compare the reliability of the photometric decomposition results of both cases (which should be identical) and the dependence of our method to derive the intrinsic shape of bulges on the galaxy inclination. We analyzed the mock images of the simulated lenticular remnants as if they were real by performing a photometric decomposition with GASP2D. We modeled the surface brightness of the bulge with a Sérsic law (Sérsic 1968), the surface brightness of the disk either with a single exponential (Freeman 1970) or with a double-exponential law (van der Kruit 1979), and the surface brightness of the bar with a Ferrers law (Ferrers 1877; Aguerri et al. 2009). An example of the GASP2D photometric decomposition of the mock images of the simulated galaxies is shown in Fig. 3 for the lenticular remnant resulting from the merger experiment gS0dE0o100 seen at an inclination $\theta=60^{\circ}$.

We listed the more relevant best-fitting structural parameters (i.e., the effective radius $r_{\mathrm{e}}$, Sérsic index $n$, and axial ratio $q_{\mathrm{b}}$ of the bulge, the scale-length $h$ and axial ratio $q_{\mathrm{d}}$ of the disk, and the difference between the position angles of bulge and disk $\delta$ ) of the mock images of the simulated lenticular remnants seen at different inclinations in Table A.1 for the giant-giant galaxy mergers, Table A. 2 for the giant-dwarf galaxy mergers, and Table A.3 for the giant S0-dwarf E0 galaxy mergers with different orbital parameters. Following Méndez-Abreu et al. (2017), we adopted $\sigma_{q}=0.01$ and $\sigma_{\mathrm{PA}}=1^{\circ}$ as uncertainties on the axial ratio and position angle of both bulge and disk, respectively.

\subsection{Bulge intrinsic shape of the simulated lenticular remnants seen at different inclinations}

We made use of our statistical method to retrieve from the mock images the probability distribution of the intrinsic axial ratios $B / A$ and $C / A$ of the bulges of the simulated lenticular remnants seen at different inclinations $\left(\theta=0^{\circ}, 30^{\circ}, 45^{\circ}, 60^{\circ}\right.$, and $\left.180^{\circ}\right)$. We excluded from the analysis the edge-on configurations $(\theta=$ $90^{\circ}$ ) because they do not allow us to constrain $B / A$ and $C / A$ due to the unknown orientation of the triaxial bulge in the disk plane.

The probability distribution of $B / A$ and $C / A$ for the bulge of the simulated lenticular remnants resulting from the merger experiment gS0dE0o100 and seen at an inclination $\theta=60^{\circ}$ is shown as an example in Fig. 4.

The face-on configurations of the simulated lenticular remnants $\left(\theta=0^{\circ}\right.$ and $\left.180^{\circ}\right)$ provided the same result in terms of the probability distribution of $B / A$ and $C / A$ for all the simulated bulges, as expected if no observational and theoretical bias affected the adopted statistical method. Furthermore, we confirmed that the tightest constraints for $B / A$ are given when galaxies are seen face on, whereas $C / A$ remains unconstrained for these galaxies.

We also found consistent probability distributions of $B / A$ and $C / A$ for the same simulated lenticular remnant seen at intermediate inclinations $\left(\theta=30^{\circ}, 45^{\circ}\right.$, and $\left.60^{\circ}\right)$. Because of this, we were confident of having correctly recovered the bulge intrinsic shape and suggested us to set a limit on the inclination of real galaxies $\left(25^{\circ}<\theta<65^{\circ}\right)$ to robustly apply our statistical method to their bulges (see Sect. 2). In general, the probability distribution of $B / A$ and $C / A$ is tighter at $\theta=60^{\circ}$ with respect to $\theta=30^{\circ}$ or $45^{\circ}$. Therefore, we considered this inclination as 

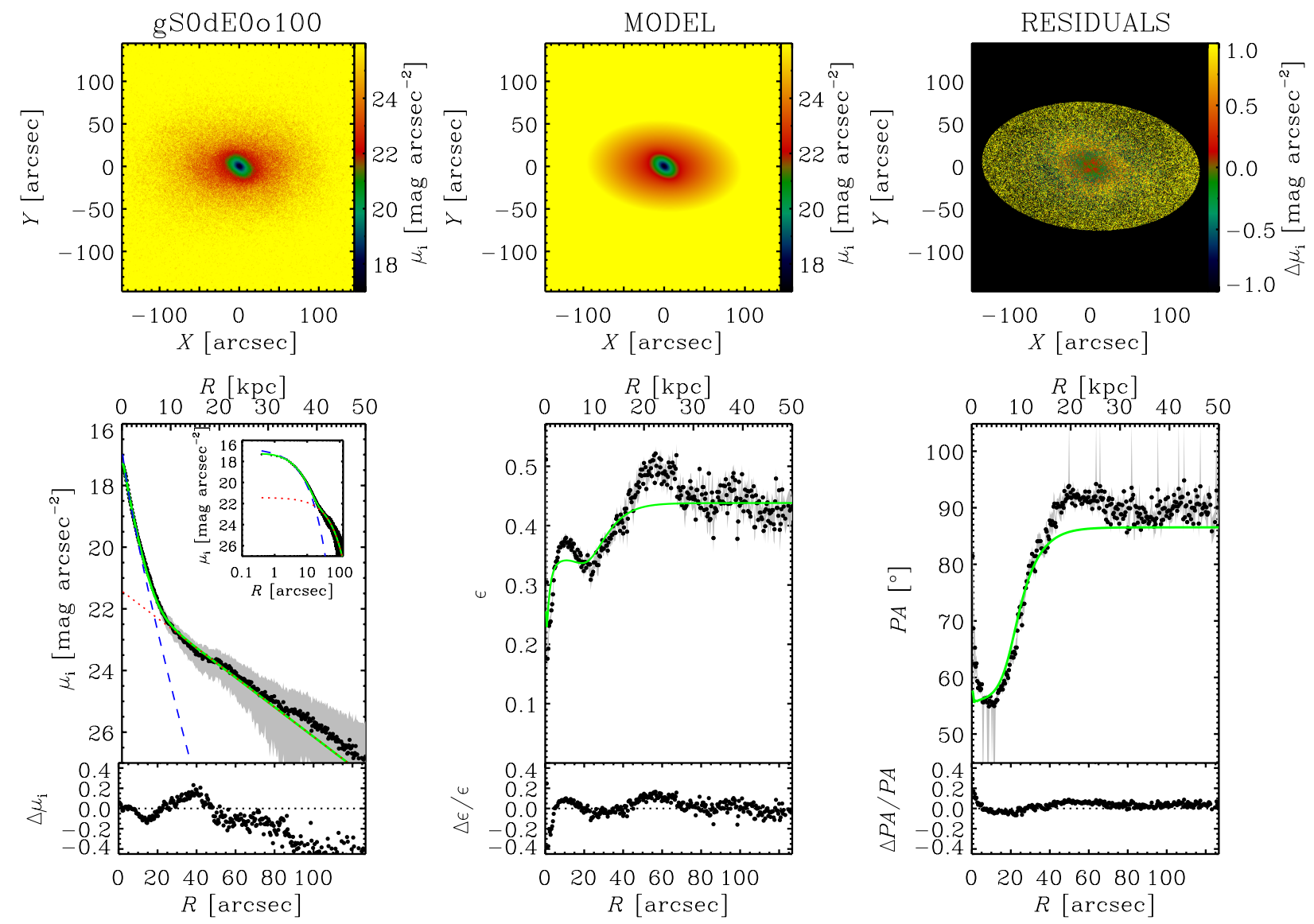

Fig. 3. Two-dimensional photometric decomposition of the mock $i$-band image of the simulated lenticular remnant gS0dE0o100 seen at an inclination $\theta=60^{\circ}$ obtained with GASP2D. The upper panels (from left to right) show the map of the observed, modeled, and residual (observed-modeled) surface brightness distributions. The lower panels (from left to right) show the ellipse-averaged radial profile of surface brightness, ellipticity, and position angle measured in the observed (black dots with gray error bars) and seeing-convolved modeled image (green solid line) and their corresponding difference. The intrinsic surface-brightness radial profiles of the best-fitting bulge (blue dashed line) and disk (red dotted line) are also shown in both linear and logarithmic scale for the distance to the center of the galaxy.

the ideal viewing angle for future analyses of the bulge intrinsic shape in real galaxies.

The probability distributions of $B / A$ and $C / A$ of the bulge of the simulated lenticular remnant resulting from the merger experiment gS0dE0o100 seen at different inclinations are shown in Fig. 5, while the remaining galaxies are in Fig. B.1. The values of $B / A$ and $C / A$ derived for all the simulated lenticular remnants seen at different inclinations are listed in Table 1.

We realized that the accuracy of the photometric decomposition is critical to successfully constrain the bulge intrinsic shape. All the structural parameters of the bulge, disk, and bar in the photometric decomposition of the mock images with GASP2D were left free to vary. For a few galaxies, we found that the $1 \sigma$ level contours of the probability distributions of $B / A$ and $C / A$ obtained at different inclinations did not overlap. We double checked the photometric decomposition of these galaxies and noticed that the ellipticity and/or position angle of their disks were not well fit by the model. As a matter of fact, we fit the surface brightness distribution of all these disks with a doubleexponential profile and assumed they had the same ellipticity and position angle both in the inner and outer regions. We found that in some cases (e.g., gE0gSbo5) the change in the ellipticity and position angle measured at the break radius was probably due to the fact that there were two distinct structural components (i.e., a lens and a disk) with different geometrical parameters, instead of a single down- or up-bending exponential disk. As a consequence, the adopted photometric model did not exquisitely match the surface brightness distribution of the simulated lenticular remnant. In other cases (e.g., gSbgSdo5), the change was due a moderate degree of granularity observed in the mock images at large galactocentric distances caused by light spots coming from isolated group of stellar particles orbiting the galaxy outskirts. To address these issues, we refined the estimate of $\epsilon_{\mathrm{d}}$ and $P A_{\mathrm{d}}$ by assuming the average ellipticity and position angle of the galaxy isophotes fit at large radii with the $\operatorname{IRAF}^{3}$ task ellipse (nominal values in Table A.1).

\section{Bulge intrinsic shape of our CALIFA galaxies}

We made use of our statistical method to retrieve the probability distribution of $B / A$ and $C / A$ of the bulges of our CALIFA galaxy sample. The probability distribution of $B / A$ and $C / A$ for the bulge of NGC 1 is shown as an example in Fig. 6, while the most probable values of $B / A$ and $C / A$ of our CALIFA bulges in Fig. 7 and Tables C.1 and C.2.

We derived for each of our CALIFA bulges the projection of the $1 \sigma$ contour along the $B / A$ and $C / A$ axes and adopted the

3 The Image Reduction and Analysis Facility is distributed by the National Optical Astronomy Observatory (NOAO), which is operated by the Association of Universities for Research in Astronomy (AURA), Inc. under cooperative agreement with the National Science Foundation. 


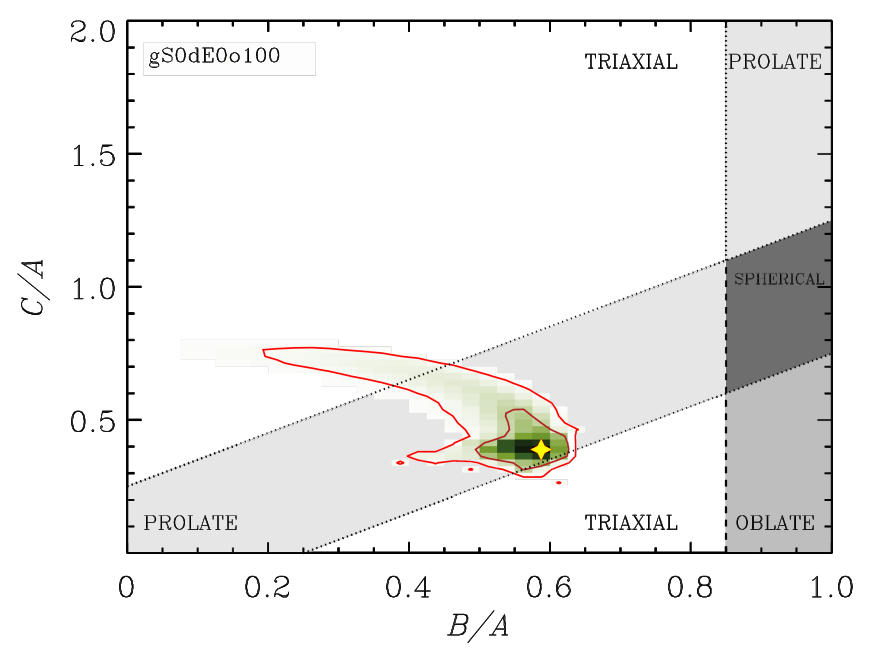

Fig. 4. Distribution of the intrinsic axial ratios $B / A$ and $C / A$ of the bulge of the simulated lenticular remnant gS0dE0o100 seen at an inclination $\theta=60^{\circ}$. The yellow star corresponds to the most probable values of $B / A$ and $C / A$. The inner and outer red solid contours encompass respectively the $68.3 \%$ and $95.4 \%$ of the realizations of $(B / A, C / A)$ consistent with the geometric parameters of bulge and disk measured from our photometric decomposition of the mock image of the simulated lenticular remnant. The white, light gray, gray, and dark gray regions mark the regimes of triaxial, prolate, oblate, and spherical bulges, respectively.

median values of such projections as the uncertainties on the derived values of $B / A$ and $C / A$. We estimated $\sigma_{B / A}=0.15$ and $\sigma_{C / A}=0.25$, respectively. At this point, we considered as oblate spheroids all the bulges with $B / A>0.85$ and $C / A<B / A-0.25$ (oblate in-plane) or with $B / A<0.85$ and $0.75<C / A<1.25$ (oblate off-plane), as prolate spheroids all the bulges with both $B / A<0.85$ and $B / A-0.25<C / A<B / A+0.25$ (prolate in-plane) or with $B / A>0.85$ and $C / A>B / A+0.25$ (prolate off-plane), as spherical all the bulges with both $B / A>0.85$ and $B / A-0.25<C / A<B / A+0.25$, and as triaxial all the remaining bulges. Spherical bulges will be treated as oblate spheroids in the analysis below.

It is worth noting that four of our CALIFA bulges are oblate spheroids off-plane, while there are none prolate spheroids offplane. Such rare central structures swelling out the disk plane have been recently studied by Corsini et al. (2012), who found a slightly triaxial polar bulge with axial ratios $B / A=0.95$ and $C / A=1.60$ in NGC 4698. We inspected the probability distribution of our 4 bulges and found that they presented a great scatter compatible also with being triaxial, as expected. Therefore, due to the peculiarity and the great uncertainty in the properties and formation mechanisms of polar bulges, they should be considered as a particular kind of bulges and not include them in the main groups described in the forthcoming analysis. Thus, the final sample of our CALIFA bulges comprises 79 objects (41 in unbarred galaxies and 38 in barred galaxies).

We distinguished all different bulges intrinsic shapes (oblate, prolate, or triaxial) in the new $(B / A, C / A)$ diagram according to the properties of their host galaxies. As a general behavior, we found that most of our CALIFA bulges tend to be oblate $(66 \%)$, with a smaller fraction of prolate $(19 \%)$ or triaxial bulges $(15 \%)$. The majority of triaxial bulges are in barred galaxies $(75 \%)$. The $B / A$ and $C / A$ distribution peaks at $\langle B / A\rangle=0.85$ and $\langle C / A\rangle=$ 0.55 , respectively.

We divided our CALIFA bulges according to their Sérsic index in the bins $n \leq 1.5,1.5<n \leq 2.5$, and $n>2.5$ (Fig. 8). The vast majority of our bulges $(80 \%)$ is characterized by a small

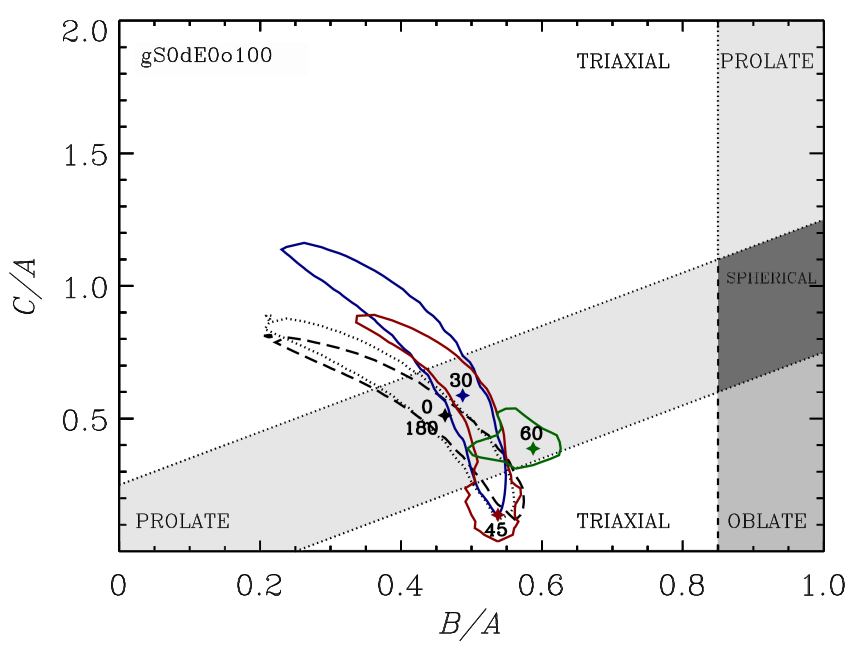

Fig. 5. Distribution of the intrinsic axial ratios $B / A$ and $C / A$ of the bulge of the simulated lenticular remnant gS0dE0o100 seen at different inclinations. The contours encompass the $68.3 \%$ of the realizations of $(B / A, C / A)$ consistent with the geometric parameters of bulge and disk measured from our photometric decomposition of the mock images of the simulated lenticular remnant at $\theta=0^{\circ}$ (black dashed line), $30^{\circ}$ (blue), $45^{\circ}$ (red), $60^{\circ}$ (green), and $180^{\circ}$ (black dotted line). The stars correspond to the most probable values of $B / A$ and $C / A$ for the different galaxy inclinations and are color coded as their corresponding contours. The white, light gray, gray, and dark gray regions mark the regimes of triaxial, prolate, oblate, and spherical bulges, respectively.

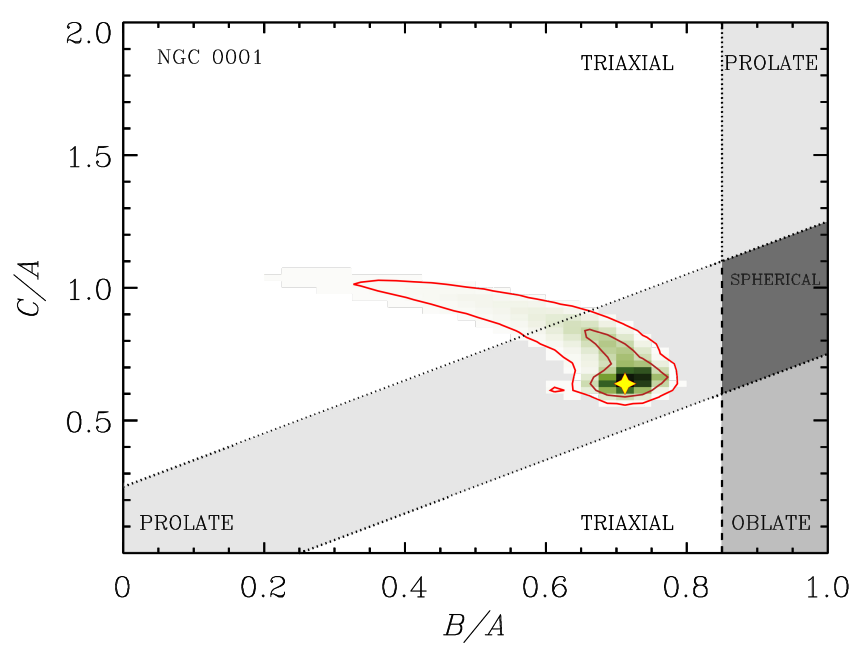

Fig. 6. As in Fig. 4, but for the bulge of NGC 1. The inner and outer red solid contours encompass respectively the $68.3 \%$ and $95.4 \%$ of the realizations of $(B / A, C / A)$ consistent with the geometric parameters of bulge and disk measured by Méndez-Abreu et al. (2017) with a photometric decomposition of the SDSS $i$-band image of the galaxy.

Sérsic index $(n \leq 2.5)$. A substantial fraction of bulges with $n>2.5(69 \%)$ is observed in unbarred galaxies. The bulges with $n \leq 1.5$ have a variety of intrinsic shapes, with comparable fractions of triaxial (30\%), oblate (49\%), and prolate bulges $(21 \%)$. By contrast, most of the bulges with $1.5<n \leq 2.5(77 \%)$ and $n>2.5(81 \%)$ are oblate. Finally, we noticed that almost all the triaxial bulges show very small values of Sérsic index $(n<1.5)$. The same trends were seen by dividing our CALIFA bulges in the bins $B / T \leq 0.1,0.1<B / T \leq 0.3$, and $B / T \leq 0.3$, according to the bulge-to-total luminosity ratio of their host galaxy (Fig. 9). Most of the larger bulges are oblate (74\%), while the 


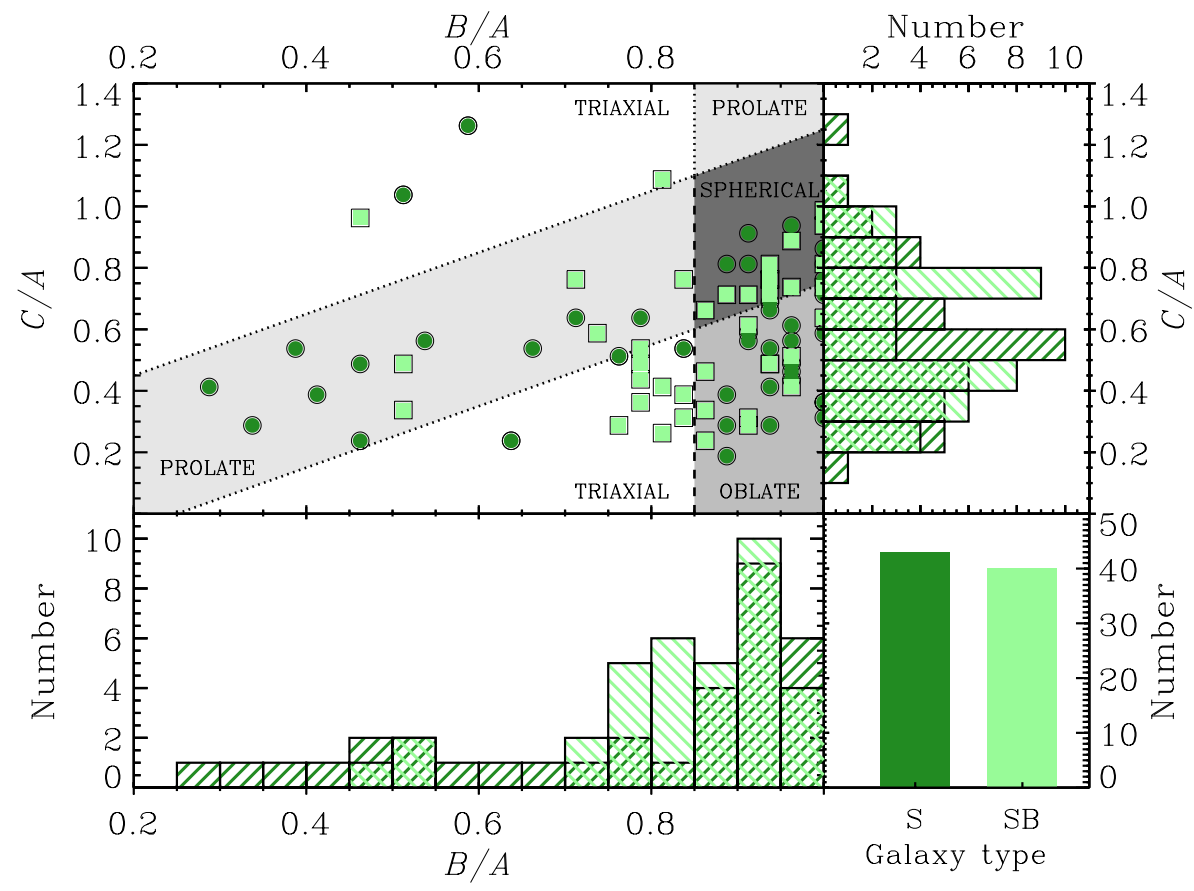

Fig. 7. Top left panel: intrinsic axial ratios $B / A$ and $C / A$ of our CALIFA bulges. Dark green circles and light green squares correspond to unbarred and barred galaxies, respectively. The white, light gray, gray, and dark gray regions mark the regimes of triaxial, prolate, oblate, and spherical bulges, respectively. Top right panel: distribution of $C / A$. Bottom left panel: distribution of $B / A$. Bottom right panel: distribution of our CALIFA bulges in unbarred (S) and barred galaxies $(\mathrm{SB})$. smaller ones show a variety of intrinsic shapes. We also pointed out that the bulges with small values of intrinsic flattening $C / A$ have systematically small values of $n$ and $B / T$.

We also analyzed the bulge intrinsic shape to highlight possible correlations with the morphology of the host galaxy (Fig. 10). We separated our CALIFA bulges into three bins by taking into account the bulges in S0 galaxies, bulges in Sa, Sab, $\mathrm{Sb}$, and $\mathrm{Sbc}$ galaxies, and bulges in $\mathrm{Sc}, \mathrm{Scd}, \mathrm{Sd}$, and $\mathrm{Sm}$ galaxies. Most of the bulges belong to galaxies in the $\mathrm{Sa}-\mathrm{Sbc}$ bin $(56 \%)$. Almost all the bulges in S0 galaxies (95\%) are oblate, with a different degree of intrinsic flattening $C / A$. We did not find any triaxial bulge among the S0 galaxies. On the contrary, the bulges of spiral galaxies present a variety of intrinsic shapes, with oblate bulges $(62 \%)$ dominating the Sa-Sbc bin. Moreover, we noticed that bulges with small values of $C / A$ are more frequently observed in late-type spirals.

Finally, we studied the bulge intrinsic shape as a function of $i$-band absolute magnitude of the bulge (Fig. 11) and of the host galaxy (Fig. 12). Almost all the most massive bulges $\left(M_{\mathrm{b}, i}<-20.5 \mathrm{mag}\right)$ are oblate $(86 \%)$, whereas the less massive one $\left(M_{\mathrm{b}, i}>-18.5 \mathrm{mag}\right)$ are more heterogeneous with a similar fraction of triaxial $(41 \%)$, oblate $(27 \%)$, and prolate systems $(32 \%)$. We obtained the same results when the total galaxy absolute magnitude was examined.

\section{Discussion}

The statistical analysis presented in this work allowed us to individually constrain the intrinsic shape of a sample of bulges in relation to their observed properties. We projected the $(B / A$, $C / A$ ) values in order to compare the bulge shape distribution with previous results (see Fig. 7). We found that the mean axial ratio of our CALIFA bulges is $\langle B / A\rangle=0.85$ and $\langle C / A\rangle=$ 0.55 , respectively. This result is in agreement with previous analyses by Bertola et al. (1991b), Fathi \& Peletier (2003), and Méndez-Abreu et al. (2008).

Since the cumulative projected distribution mixes all different shapes (oblate, prolate, and triaxial), we preferred to distinguish the properties of our CALIFA bulges in the $(B / A, C / A)$ diagram. Indeed, the actual position of bulges in the $(B / A, C / A)$ diagram is a powerful tool for disentangling bulge types. We found that some of our CALIFA bulges $(6 \%)$ are very flattened oblate systems $(B / A>0.85$ and $C / A<0.3)$, which are possible candidate to be disk-like bulges. Moreover, since barred galaxies are found to host the majority of triaxial bulges, they could be interpreted as the signature of boxy/peanut structures. Indeed, the secular evolution of the bar via buckling or resonants effects is known to result in thick triaxial components (Athanassoula 2005; Martinez-Valpuesta et al. 2006, and references in Sect. 1). Even the inclusion of the bar in the photometric decomposition can not avoid a mild contamination from boxy/peanut structures. Thus, it is not surprising that barred galaxies show a large fraction of triaxial bulges. It is worth noting that in discussing the shape of the sample bulges obtained from the $(B / A, C / A)$ diagram, we considered the statistical meaning of the intrinsic axial ratios that we derived and the empiric definition we adopted for the oblate, spherical, prolate, and triaxial bulges. The $1 \sigma$ contour level of the distribution of the intrinsic axial ratios of a bulge can tightly or loosely circle the most probable values of its shape (see Fig. 5, for an example). Therefore, a certain degree of triaxiality is allowed also for the bulges we classified as oblate or prolate. On the other hand, the definition of the boundaries of the regions marking the different bulge shapes in the $(B / A, C / A)$ diagram might be very conservative.

These results are consistent with a major role of a certain mechanism in the buildup of the most massive bulges (usually identified by higher $n$, higher $B / T$, earlier types, more massive systems) which has not significantly contributed to those forming the less massive ones (usually identified by lower $n$, lower $B / T$, later types and in less massive galaxies). Some evolutionary mechanisms may have taken place in all mass ranges similarly (such as internal secular evolution or cluster-related processes), but it is obvious from these results that there are some specific processes that have contributed much more in the most massive bulges to make them more homogeneous in shape (i.e., all oblate) than in the bulges with lower masses (e.g., 


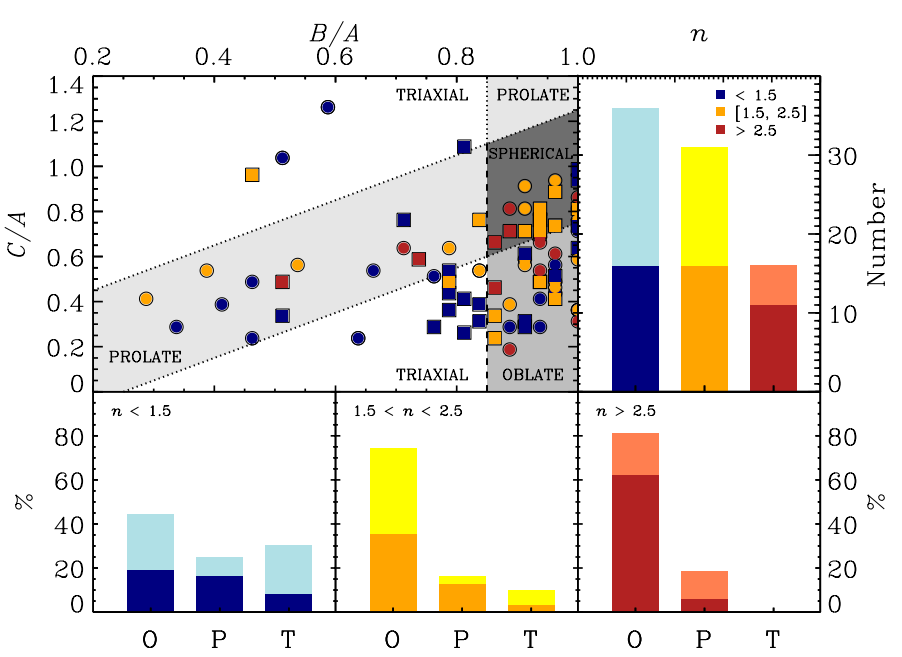

Fig. 8. Top left panel: intrinsic axial ratios $B / A$ and $C / A$ of our CALIFA bulges as a function of their Sérsic index $(n<1.5$ : blue symbols; $1.5<n<2.5$ : yellow symbols; $n>2.5$ : red symbols). Circles and squares correspond to unbarred and barred galaxies, respectively. The white, light gray, gray, and dark gray regions mark the regimes of triaxial, prolate, oblate, and spherical bulges, respectively. Top right panel: distribution of the Sérsic index of our CALIFA bulges $(n<1.5$ : blue histogram; $1.5<n<2.5$ : yellow histogram; $n>2.5$ : red histogram). Dark and light colors correspond to unbarred and barred galaxies, respectively. Bottom panels: distribution of the intrinsic shape of our CALIFA bulges (O: oblate; $\mathrm{P}$ : prolate; $\mathrm{T}$ : triaxial) as a function of their Sérsic index $(n<1.5$ : blue histograms; $1.5<n<2.5$ : yellow histograms; $n>2.5$ : red histograms). Dark and light colors correspond to unbarred and barred galaxies, respectively.

Ravikumar et al. 2006; Laurikainen et al. 2010; Bernardi et al. 2011a,b). These processes must impose over others and have occurred more frequently in massive systems than in less massive ones, as well as they have also contributed to increase $n$ and $B / T$ in the galaxy at the same time, meaning that they must transform the system toward an earlier type.

In the less massive bulges, the interplay of different evolutionary mechanisms can explain the wide variety of shapes, as they can be more or less relevant in a galaxy depending on its evolutionary history and environment (Buta et al. 2010; Marino et al. 2011). However, the presence of the bar seems to drive the evolution of low-mass triaxial bulges. Indeed, triaxial bulges are mostly hosted in barred galaxies with low values of $B / T, M_{\mathrm{b}, i}$, and $n$. These bulges could be contaminated by the residual light of the low-inclined counterparts of boxy/peanut structures. The lack of triaxial bulges in lenticular barred galaxies could be explained by the larger mass of their bulges: their deep potential well seems to reshape the central region into a more axisymmetric structure, where the bar has a marginal role in perturbing the bulge. Thus, the bulge mass could play a role also in driving the evolution of bulges in barred galaxies.

Many studies report observational evidence of a major role of both major and minor merging and dissipative collapse in the buildup of the most massive galaxies (e.g., Rudick et al. 2009; Eliche-Moral et al. 2010; Thomas et al. 2010; Kaviraj et al. 2011; Bernardi et al. 2011b; Méndez-Abreu et al. 2012; Barway et al. 2013; Prieto et al. 2013; Morelli et al. 2016; Leja et al. 2015; Prieto \& Eliche-Moral 2015). Numerical simulations have shown that gas-poor major and minor mergers tend to introduce some triaxiality in bulges that originally were spheroidal (Cox et al. 2006; Tapia et al. 2014). However, the bulges of dry minor-merger remnants also exhibit higher rotational support at their centers, even though the global rotational

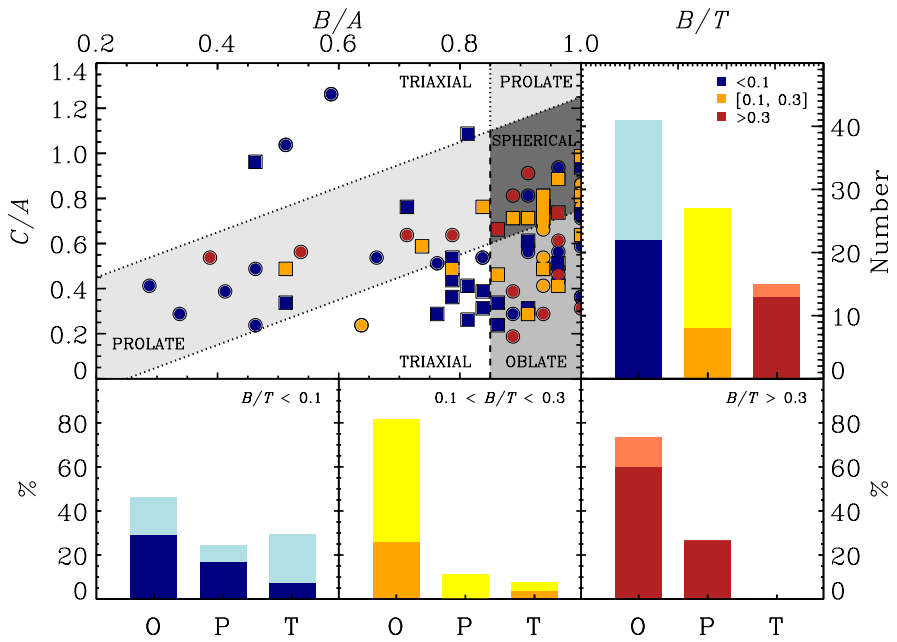

Fig. 9. As in Fig. 8, but for the bulge-to-total luminosity ratio. Our CALIFA bulges are divided in the following bins: $B / T>0.1$ (blue), $0.1<B / T<0.3$ (yellow), and $B / T>0.3$ (red).

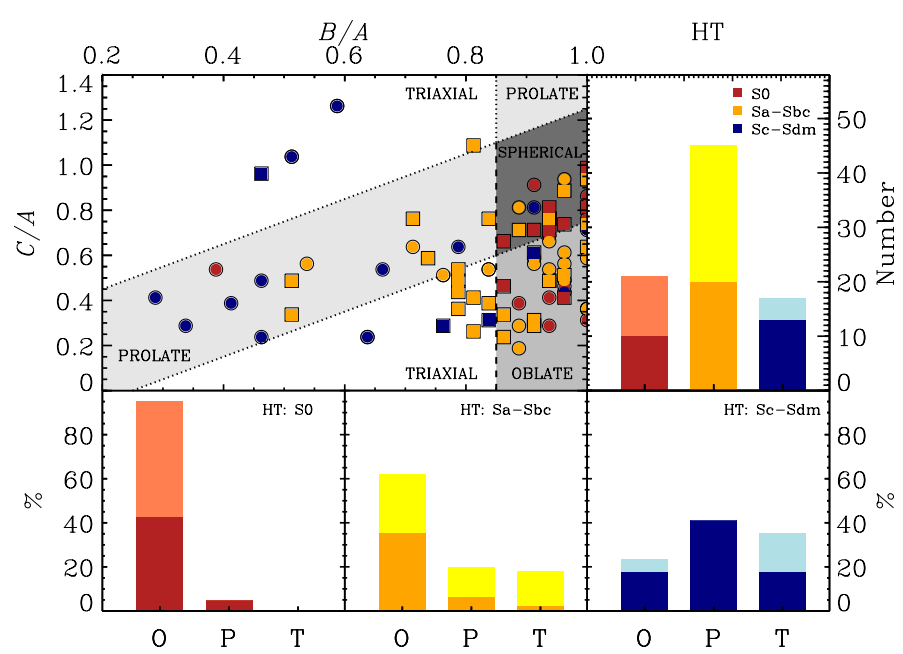

Fig. 10. As in Fig. 8, but for the Hubble type. Our CALIFA bulges are divided in the following bins: S0 (red), Sa-Sbc (yellow), Sc-Sdm (blue).

support of the galaxy decreases, making the bulge more oblate (Tapia et al. 2014). This happens because part of the orbital angular momentum of the encounter is transferred to the inner regions (see Eliche-Moral et al. 2006, 2011), contributing to the flattening of the material at the galaxy center. High gas amounts in the progenitors only contribute to make the remnant more axisymmetric (Jesseit et al. 2007), so the trend of dry mergers to make remnant bulges more oblate can be extrapolated to wet ones. Therefore, our results would be consistent with a higher relevance of merging in the formation and evolution of the most massive bulges.

\section{Conclusions}

We derived the intrinsic shape of 83 bulges of a sample of nearby galaxies from CALIFA DR3. To this aim we applied the statistical method by Méndez-Abreu et al. (2008) to the structural parameters obtained by Méndez-Abreu et al. (2017) with a twodimensional photometric decomposition of the SDSS $i$-band images of the sample galaxies. 


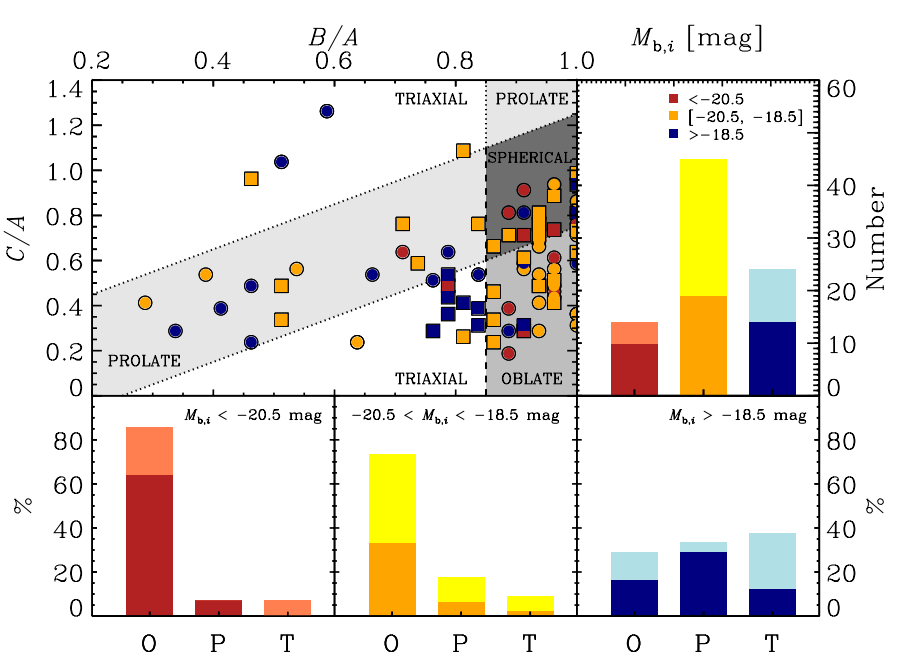

Fig. 11. As in Fig. 8, but for the absolute magnitude of the bulge. Our CALIFA bulges are divided in the following bins: $M_{\mathrm{b}, i}<-20.5$ mag (red), $-20.5<M_{\mathrm{b}, i}<-18.5 \mathrm{mag}$ (yellow), and $M_{\mathrm{b}, i}>-18.5 \mathrm{mag}$ (blue).

We made use of a set of simulated galaxies resulting from merger experiments, that closely resembling lenticular galaxies, to test the reliability of the method by Méndez-Abreu et al. (2008). For each simulated lenticular remnant, we created a set of mock SDSS $i$-band images at different galaxy inclinations to mimic the observing setup of SDSS images of CALIFA DR3 galaxies. We performed a two-dimensional photometric decomposition of all the mock images applying the same procedure as for real galaxies, in order to retrieve the geometrical parameters of bulge and disk which we used to recover the bulge intrinsic shape. The probability distributions of the axial ratios $B / A$ and $C / A$ obtained for different inclinations for the same simulated lenticular remnant overlap at $1 \sigma$ level. We concluded that the adopted method allows us to successfully constrain the bulge intrinsic shape when the galaxy inclination is $25^{\circ}<\theta<65^{\circ}$. We also realized that a very accurate photometric decomposition is mandatory to retrieve the bulge intrinsic shape and that a galaxy inclination of $\theta=60^{\circ}$ returns the tightest constraints on the intrinsic axial ratios $B / A$ and $C / A$ of the bulge.

We divided our CALIFA bulges according to their intrinsic axial ratios $B / A$ and $C / A$ into oblate (in-plane or off-plane), prolate (in-plane or off-plane), and triaxial. We looked for possible correlations between the intrinsic shape of our bulges and some of the basic properties of their host galaxies (i.e., Sérsic index of the bulge $n$, bulge-to-total luminosity ratio $B / T, H u b$ ble type HT, $i$-band absolute magnitude of the bulge $M_{\mathrm{b}, i}$, and $i$-band absolute magnitude of the galaxy $M_{i}$ ) as derived by Méndez-Abreu et al. 2017. Our analysis pointed out that bulges with a small value of $n$ or $B / T$ could be equally axisymmetric or triaxial ellipsoids, while most of the bulges with large values of $n$ or $B / T$ are mostly oblate spheroids. Moreover, less massive bulges and bulges in late-type galaxies presented heterogeneous intrinsic shapes, while more massive bulges and bulges in lenticular galaxies are mostly oblate. Finally, we found the majority of triaxial bulges in barred galaxies.

We concluded that merging events and dissipative collapse could be responsible of driving the formation and evolution of our most massive bulges, although other physical mechanisms, that is, the internal secular evolution caused by the presence of the bar, may be acting at the same time. The coexistence of different pathways is more clear in less massive bulges, where the

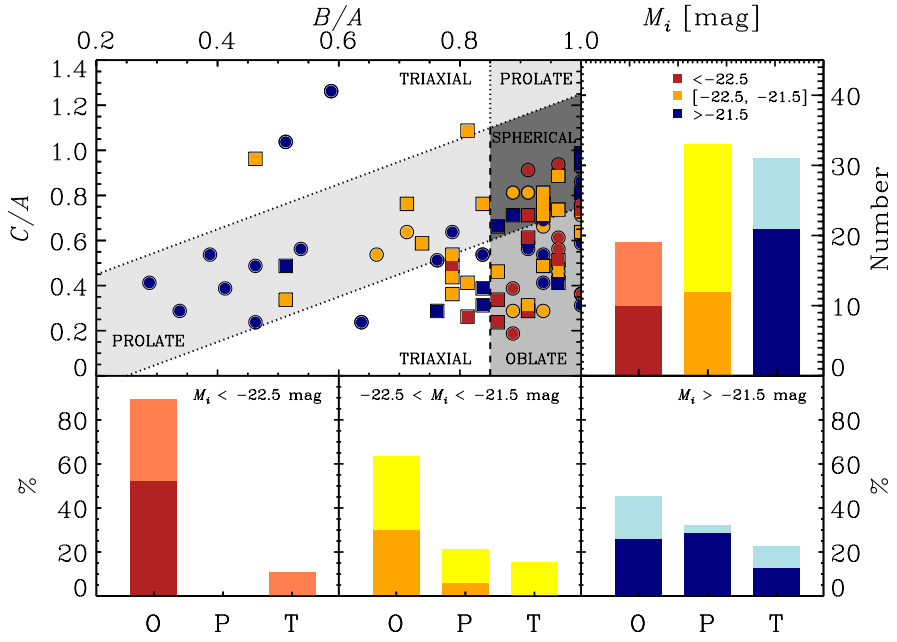

Fig. 12. As in Fig. 8, but for the absolute magnitude of the galaxy. Our CALIFA bulges are divided in the following bins: $M_{i}<-22.5 \mathrm{mag}$ (red), $-22.5<M_{i}<-21.5 \mathrm{mag}$ (yellow), and $M_{i}>-21.5 \mathrm{mag}$ (blue).

bar seems to reshape low-mass triaxial bulges. In this context, the role of simulations result crucial in unveiling various evolution pathways in nearby galaxies. Nevertheless, very few numerical studies have focused on the bulge evolution and in particular on the intrinsic shape. Thus, our results have imposed further limitations on forthcoming numerical simulations and may help to disentangle different formation scenarios.

Acknowledgements. We would like to thank the anonymous referee for the suggestions that helped us to improve the way we presented our results. This work is supported by Padua University through grants 60A02-4434/15, DOR1699945, and DOR1715817. E.M.C. and L.M. acknowledge financial support from Padua University through grants BIRD164402/16 an CPS0204, respectively. J.M.A. want to thank the support of this work by the Spanish Ministerio de Economia y Competitividad (MINECO) under the grant AYA2013-43188-P. L.C. thanks the IAC for hospitality while this paper was in progress. We would also like to acknowledge I. Chilingarian, P. Di Matteo, F. Combes, A.-L. Melchior, and B. Semelin for creating the GalMer database, and the HORIZON project for supporting it (http://www.projet-horizon.fr/rubrique3.html). This research made use of Sloan Digital Sky Survey (SDSS) data (http://www.sdss.org) and NASA/IPAC Extragalactic Database (NED) (http://ned.ipac.caltech.edu/).

\section{References}

Abazajian, K. N., Adelman-McCarthy, J. K., Agüeros, M. A., et al. 2009, ApJS, 182,543

Aguerri, J. A. L., Balcells, M., \& Peletier, R. F. 2001, A\&A, 367, 428

Aguerri, J. A. L., Méndez-Abreu, J., \& Corsini, E. M. 2009, A\&A, 495, 491

Andredakis, Y. C., Peletier, R. F., \& Balcells, M. 1995, MNRAS, 275, 874

Athanassoula, E. 2005, MNRAS, 358, 1477

Athanassoula, E., \& Beaton, R. L. 2006, MNRAS, 370, 1499

Balcells, M., Graham, A. W., \& Peletier, R. F. 2007, ApJ, 665, 1104

Barway, S., Wadadekar, Y., Vaghmare, K., \& Kembhavi, A. K. 2013, MNRAS, 432, 430

Benítez, E., Méndez-Abreu, J., Fuentes-Carrera, I., et al. 2013, ApJ, 763, 136

Bernardi, M., Roche, N., Shankar, F., \& Sheth, R. K. 2011a, MNRAS, 412, 684

Bernardi, M., Roche, N., Shankar, F., \& Sheth, R. K. 2011b, MNRAS, 412, L6 Bertelli, G., Bressan, A., Chiosi, C., Fagotto, F., \& Nasi, E. 1994, A\&AS, 106

Bertola, F., \& Capaccioli, M. 1975, ApJ, 200, 439

Bertola, F., \& Galletta, G. 1979, A\&A, 77, 363

Bertola, F., Bettoni, D., Danziger, J., et al. 1991a, ApJ, 373, 369

Bertola, F., Vietri, M., \& Zeilinger, W. W. 1991b, ApJ, 374, L13

Borlaff, A., Eliche-Moral, M. C., Rodríguez-Pérez, C., et al. 2014, A\&A, 570, A103 
Bournaud, F. 2016, in Galactic Bulges, eds. E. Laurikainen, R. Peletier, \& D. Gadotti (Berlin: Springer), 355

Brooks, A., \& Christensen, C. 2016, in Galactic Bulges, eds. E. Laurikainen, R. Peletier, \& D. Gadotti (Berlin: Springer), 317

Bruzual, G., \& Charlot, S. 2003, MNRAS, 344, 1000

Buta, R., Laurikainen, E., Salo, H., \& Knapen, J. H. 2010, ApJ, 721, 259

Carter, D. 1978, MNRAS, 182, 797

Ceverino, D., Dekel, A., Tweed, D., \& Primack, J. 2015, MNRAS, 447, 3291

Chabrier, G. 2003, PASP, 115, 763

Chilingarian, I. V., Di Matteo, P., Combes, F., Melchior, A.-L., \& Semelin, B. 2010, A\&A, 518, A61

Coelho, P., \& Gadotti, D. A. 2011, ApJ, 743, L13

Combes, F., \& Sanders, R. H. 1981, A\&A, 96, 164

Corsini, E. M., Méndez-Abreu, J., Pastorello, N., et al. 2012, MNRAS, 423, L79

Costantin, L., Méndez-Abreu, J., Corsini, E. M., et al. 2017, A\&A, 601, A84

Cox, T. J., Dutta, S. N., Di Matteo, T., et al. 2006, ApJ, 650, 791

Dekel, A., Sari, R., \& Ceverino, D. 2009, ApJ, 703, 785

Di Matteo, P., Combes, F., Melchior, A.-L., \& Semelin, B. 2007, A\&A, 468, 61

Di Matteo, P., Bournaud, F., Martig, M., et al. 2008, A\&A, 492, 31

Eggen, O. J., Lynden-Bell, D., \& Sandage, A. R. 1962, ApJ, 136, 748

Eliche-Moral, M. C., Balcells, M., Aguerri, J. A. L., \& González-García, A. C. 2006, A\&A, 457, 91

Eliche-Moral, M. C., Prieto, M., Gallego, J., et al. 2010, A\&A, 519, A55

Eliche-Moral, M. C., González-García, A. C., Balcells, M., et al. 2011, A\&A, 533, A 104

Erwin, P. 2015, ApJ, 799, 226

Erwin, P., \& Debattista, V. P. 2013, MNRAS, 431, 3060

Erwin, P., Saglia, R. P., Fabricius, M., et al. 2015, MNRAS, 446, 4039

Fathi, K., \& Peletier, R. F. 2003, A\&A, 407, 61

Ferrers, N. M. 1877, Quart. J. Pure Appl. Math., 14, 1

Freeman, K. C. 1970 , ApJ, 160, 811

Gadotti, D. A. 2009, MNRAS, 393, 1531

Hopkins, P. F., Kereš, D., Murray, N., Quataert, E., \& Hernquist, L. 2012, MNRAS, 427, 968

Illingworth, G. 1977, ApJ, 218, L43

Jesseit, R., Naab, T., Peletier, R. F., \& Burkert, A. 2007, MNRAS, 376, 997

Kauffmann, G. 1996, MNRAS, 281, 487

Kaviraj, S., Tan, K.-M., Ellis, R. S., \& Silk, J. 2011, MNRAS, 411, 2148

Kormendy, J. 2016, in Galactic Bulges, eds. E. Laurikainen, R. Peletier, \&

D. Gadotti (Berlin: Springer), 431

Kormendy, J., \& Kennicutt, Jr., R. C. 2004, ARA\&A, 42, 603

Krajnović, D., Emsellem, E., Cappellari, M., et al. 2011, MNRAS, 414, 2923

Laurikainen, E., Salo, H., \& Buta, R. 2005, MNRAS, 362, 1319

Laurikainen, E., Salo, H., Buta, R., Knapen, J. H., \& Comerón, S. 2010 MNRAS, 405, 1089

Laurikainen, E., Salo, H., Athanassoula, E., Bosma, A., \& Herrera-Endoqui, M. 2014, MNRAS, 444, L80

Leja, J., van Dokkum, P. G., Franx, M., \& Whitaker, K. E. 2015, ApJ, 798, 115 Lindblad, B. 1956, Stockholm Obs. Ann., 19, 7

Lotz, J. M., Jonsson, P., Cox, T. J., \& Primack, J. R. 2008, MNRAS, 391, 1137

Lütticke, R., Dettmar, R.-J., \& Pohlen, M. 2000, A\&A, 362, 435

Marino, A., Bianchi, L., Rampazzo, R., et al. 2011, ApJ, 736, 154

Martinez-Valpuesta, I., Shlosman, I., \& Heller, C. 2006, ApJ, 637, 214
Méndez-Abreu, J. 2016, in Galactic Bulges, eds. E. Laurikainen, R. Peletier, \& D. Gadotti (Berlin: Springer), 15

Méndez-Abreu, J., Aguerri, J. A. L., Corsini, E. M., \& Simonneau, E. 2008, A\&A, 478, 353

Méndez-Abreu, J., Simonneau, E., Aguerri, J. A. L., \& Corsini, E. M. 2010, A\&A, 521, A71

Méndez-Abreu, J., Aguerri, J. A. L., Barrena, R., et al. 2012, A\&A, 537, A25

Méndez-Abreu, J., Debattista, V. P., Corsini, E. M., \& Aguerri, J. A. L. 2014 A\&A, 572, A25

Méndez-Abreu, J., Ruiz-Lara, T., Sánchez-Menguiano, L., et al. 2017, A\&A, 598, A32

Mihos, J. C., \& Hernquist, L. 1994, ApJ, 437, 611

Moffat, A. F. J. 1969, A\&A, 3, 455

Morelli, L., Pompei, E., Pizzella, A., et al. 2008, MNRAS, 389, 341 Morelli, L., Parmiggiani, M., Corsini, E. M., et al. 2016, MNRAS, 463, 4396

Naab, T., Oser, L., Emsellem, E., et al. 2014, MNRAS, 444, 3357

Oser, L., Naab, T., Ostriker, J. P., \& Johansson, P. H. 2012, ApJ, 744, 63

Peng, C. Y., Ho, L. C., Impey, C. D., \& Rix, H.-W. 2002, AJ, 124, 266

Prieto, M., \& Eliche-Moral, M. C. 2015, MNRAS, 451, 1158

Prieto, M., Eliche-Moral, M. C., Balcells, M., et al. 2013, MNRAS, 428, 999

Querejeta, M., Eliche-Moral, M. C., Tapia, T., et al. 2015a, A\&A, 573, A78

Querejeta, M., Eliche-Moral, M. C., Tapia, T., et al. 2015b, A\&A, 579, L2

Ravikumar, C. D., Barway, S., Kembhavi, A., Mobasher, B., \& Kuriakose, V. C. 2006, A\&A, 446, 827

Rodríguez, S., \& Padilla, N. D. 2013, MNRAS, 434, 2153

Rudick, C. S., Mihos, J. C., Frey, L. H., \& McBride, C. K. 2009, ApJ, 699, 1518

Ryden, B. S. 2004, ApJ, 601, 214

Ryden, B. S. 2006, ApJ, 641, 773

Sánchez, S. F., García-Benito, R., Zibetti, S., et al. 2016, A\&A, 594, A36

Sandage, A. 1990, JRASC, 84, 70

Sandage, A., Freeman, K. C., \& Stokes, N. R. 1970, ApJ, 160, 831

Semelin, B., \& Combes, F. 2002, A\&A, 388, 826

Sérsic, J. L. 1968, Atlas de Galaxias Australes (Cordoba: Observatorio Astronomico de Cordoba)

Sil'chenko, O. K. 2013, Astron. Nachr., 334, 781

Sil'chenko, O. K., Proshina, I. S., Shulga, A. P., \& Koposov, S. E. 2012, MNRAS, 427, 790

Simonneau, E., Varela, A. M., \& Munoz-Tunon, C. 1998, Nuovo Cimento B, 113,927

Statler, T. S., Lambright, H., \& Bak, J. 2001, ApJ, 549, 871

Tapia, T., Eliche-Moral, M. C., Querejeta, M., et al. 2014, A\&A, 565, A31

Tapia, T., Eliche-Moral, M. C., Aceves, H., et al. 2017, A\&A, 604, A105

Thomas, D., Maraston, C., Schawinski, K., Sarzi, M., \& Silk, J. 2010, MNRAS, 404, 1775

Toomre, A. 1977, in Evolution of Galaxies and Stellar Populations, eds. B. M. Tinsley \& R. B. G. Larson (New Haven: Yale University Press), 401

Tremblay, B., \& Merritt, D. 1996, AJ, 111, 2243

van den Bosch, R. C. E., van de Ven, G., Verolme, E. K., Cappellari, M., \& de Zeeuw, P. T. 2008, MNRAS, 385, 647

van der Kruit, P. C. 1979, A\&AS, 38, 15

Walcher, C. J., Wisotzki, L., Bekeraité, S., et al. 2014, A\&A, 569, A1

Weijmans, A.-M., de Zeeuw, P. T., Emsellem, E., et al. 2014, MNRAS, 444, 3340

Williams, T. B., \& Schwarzschild, M. 1979, ApJ, 227, 56 
L. Costantin et al.: The intrinsic shape of bulges in the CALIFA survey

\section{Appendix A}

Table A.1. Structural parameters of the simulated lenticular remnants resulting from numerical experiments of giant-giant galaxy mergers.

\begin{tabular}{|c|c|c|c|c|c|c|c|}
\hline $\begin{array}{c}\text { Galaxy } \\
\text { (1) }\end{array}$ & $\begin{array}{c}\theta \\
{\left[{ }^{\circ}\right]} \\
(2)\end{array}$ & $\begin{array}{c}r_{\mathrm{e}} \\
\text { [arcsec] } \\
(3)\end{array}$ & (4) & $\begin{array}{c}h \\
{[\operatorname{arcsec}]} \\
(5)\end{array}$ & $\begin{array}{l}q_{\mathrm{b}} \\
(6)\end{array}$ & $\begin{array}{l}q_{\mathrm{d}} \\
(7)\end{array}$ & $\begin{array}{l}|\delta| \\
{\left[^{\circ}\right]} \\
(8)\end{array}$ \\
\hline \multirow{5}{*}{ gE0gSbo5 } & 0 & 0.8 & 3.8 & 9.0 & 0.95 & 0.98 & 90 \\
\hline & 180 & 0.8 & 3.8 & 9.0 & 1.00 & 0.98 & 32 \\
\hline & 30 & 0.9 & 4.9 & 9.1 & 1.00 & 0.97 & 19 \\
\hline & 45 & 0.9 & 3.9 & 9.4 & 0.86 & $0.78^{*}$ & 4 \\
\hline & 60 & 1.0 & 4.1 & 9.7 & 0.78 & $0.67^{*}$ & 1 \\
\hline \multirow{5}{*}{ gE0gSdo5 } & 0 & 1.0 & 1.1 & 8.7 & 0.80 & 0.99 & 6 \\
\hline & 180 & 1.0 & 1.1 & 8.7 & 0.80 & 0.99 & 6 \\
\hline & 30 & 0.9 & 1.2 & 8.7 & 0.90 & $0.90^{*}$ & 23 \\
\hline & 45 & 0.9 & 1.3 & 8.9 & 0.81 & $0.83^{*}$ & 18 \\
\hline & 60 & 0.8 & 1.5 & 9.1 & 0.64 & $0.70^{*}$ & 7 \\
\hline \multirow{5}{*}{ gSbgSbo 9} & 0 & 1.3 & 3.5 & 10.9 & 0.72 & 0.97 & 90 \\
\hline & 180 & 1.3 & 3.5 & 10.9 & 0.72 & 0.97 & 90 \\
\hline & 30 & 1.2 & 3.8 & 11.0 & 0.78 & 0.87 & 63 \\
\hline & 45 & 1.1 & 4.2 & 11.3 & 0.81 & 0.74 & 42 \\
\hline & 60 & 1.1 & 4.9 & 11.6 & 0.73 & 0.57 & 18 \\
\hline \multirow{5}{*}{ gSbgSdo5 } & 0 & 2.3 & 3.6 & 18.0 & 0.83 & 0.99 & 58 \\
\hline & 180 & 2.4 & 4.0 & 18.1 & 0.84 & 0.99 & 59 \\
\hline & 30 & 2.3 & 3.8 & 18.0 & 0.82 & 0.92 & $29^{*}$ \\
\hline & 45 & 2.2 & 3.6 & 17.4 & 0.75 & 0.82 & $21^{*}$ \\
\hline & 60 & 2.1 & 3.5 & 17.4 & 0.64 & 0.67 & $12^{*}$ \\
\hline
\end{tabular}

Notes. (1) Identifier of the simulated lenticular remnant. (2) Galaxy inclination. (3) Effective radius of the bulge. (4) Sérsic index of the bulge. (5) Scale length of the disk. (6), (7) Apparent axial ratio of the bulge and disk, respectively. (8) Difference of the position angles of bulge and disk. Nominal values are marked with *.

Table A.2. As in Table A.1, but simulated lenticular remnants resulting from numerical experiments of giant-dwarf galaxy mergers.

\begin{tabular}{|c|c|c|c|c|c|c|c|}
\hline $\begin{array}{c}\text { Galaxy } \\
\text { (1) }\end{array}$ & $\begin{array}{c}\theta \\
{\left[{ }^{\circ}\right]} \\
(2)\end{array}$ & $\begin{array}{c}r_{\mathrm{e}} \\
{[\operatorname{arcsec}]} \\
(3)\end{array}$ & $\begin{array}{l}n \\
(4)\end{array}$ & $\begin{array}{c}h \\
{[\operatorname{arcsec}]} \\
(5)\end{array}$ & $\begin{array}{l}q_{\mathrm{b}} \\
(6)\end{array}$ & $\begin{array}{l}q_{\mathrm{d}} \\
\text { (7) }\end{array}$ & $\begin{array}{l}|\delta| \\
\left.{ }^{\circ}\right] \\
(8)\end{array}$ \\
\hline \multirow{5}{*}{ gS0dS0o99 } & 0 & 7.0 & 1.1 & 18.9 & 0.60 & 0.97 & 59 \\
\hline & 180 & 7.0 & 1.1 & 19.2 & 0.60 & 0.96 & 82 \\
\hline & 30 & 6.8 & 1.1 & 18.9 & 0.59 & 0.89 & 33 \\
\hline & 45 & 6.6 & 1.1 & 18.8 & 0.57 & 0.76 & 18 \\
\hline & 60 & 6.3 & 1.1 & 18.5 & 0.53 & 0.59 & 11 \\
\hline \multirow{5}{*}{ gS0dSao103 } & 0 & 7.7 & 1.5 & 22.1 & 0.54 & 0.97 & 20 \\
\hline & 180 & 7.8 & 1.5 & 22.1 & 0.54 & 0.98 & 20 \\
\hline & 30 & 7.4 & 1.5 & 21.6 & 0.56 & 0.93 & 46 \\
\hline & 45 & 6.9 & 1.6 & 21.5 & 0.60 & 0.80 & 47 \\
\hline & 60 & 6.2 & 1.6 & 21.1 & 0.63 & 0.63 & 37 \\
\hline \multirow{5}{*}{ gS0dSdo106 } & 0 & 7.7 & 1.1 & 24.1 & 0.46 & 0.93 & 83 \\
\hline & 180 & 7.7 & 1.0 & 24.1 & 0.46 & 0.93 & 83 \\
\hline & 30 & 7.4 & 1.1 & 22.2 & 0.46 & 0.92 & 36 \\
\hline & 45 & 6.8 & 1.0 & 21.2 & 0.45 & 0.77 & 20 \\
\hline & 60 & 8.3 & 1.3 & 30.1 & 0.43 & 0.59 & 9 \\
\hline \multirow{5}{*}{ gS0dSdo100 } & 0 & 7.9 & 1.6 & 26.3 & 0.53 & 0.93 & 73 \\
\hline & 180 & 7.9 & 1.6 & 26.3 & 0.53 & 0.93 & 74 \\
\hline & 30 & 7.9 & 1.6 & 24.9 & 0.50 & 0.91 & 13 \\
\hline & 45 & 8.0 & 1.7 & 25.1 & 0.47 & 0.76 & 4 \\
\hline & 60 & 8.3 & 1.7 & 25.7 & 0.44 & 0.56 & 1 \\
\hline
\end{tabular}

Table A.3. As in Table A.1, but simulated lenticular remnants resulting from numerical experiments of (giant S0)-(dwarf E0) galaxy mergers with different orbital parameters.

\begin{tabular}{|c|c|c|c|c|c|c|c|}
\hline $\begin{array}{c}\text { Galaxy } \\
\text { (1) }\end{array}$ & $\begin{array}{c}\theta \\
{\left[{ }^{\circ}\right]} \\
(2)\end{array}$ & $\begin{array}{c}r_{\mathrm{e}} \\
\text { [arcsec] } \\
(3)\end{array}$ & (4) & $\begin{array}{c}h \\
\text { [arcsec] } \\
(5)\end{array}$ & $\begin{array}{l}q_{\mathrm{b}} \\
(6)\end{array}$ & $\begin{array}{l}q_{\mathrm{d}} \\
(7)\end{array}$ & $\begin{array}{l}|\delta| \\
{\left[{ }^{\circ}\right]} \\
(8)\end{array}$ \\
\hline \multirow{5}{*}{ gS0dE0o98 } & 0 & 7.8 & 1.1 & 21.5 & 0.49 & 0.90 & 9 \\
\hline & 180 & 7.9 & 1.1 & 21.6 & 0.49 & 0.91 & 13 \\
\hline & 30 & 7.4 & 1.1 & 21.7 & 0.52 & 0.82 & 30 \\
\hline & 45 & 6.8 & 1.2 & 21.9 & 0.54 & 0.70 & 26 \\
\hline & 60 & 6.4 & 1.2 & 22.4 & 0.54 & 0.53 & 19 \\
\hline \multirow{5}{*}{ gS0dE0o99 } & 0 & 7.8 & 1.1 & 20.8 & 0.49 & 0.99 & 16 \\
\hline & 180 & 7.8 & 1.1 & 20.7 & 0.49 & 0.97 & 16 \\
\hline & 30 & 6.7 & 1.1 & 18.5 & 0.56 & 0.91 & 7 \\
\hline & 45 & 5.9 & 1.2 & 18.5 & 0.66 & 0.89 & 54 \\
\hline & 60 & 4.9 & 1.2 & 18.7 & 0.76 & 0.66 & 40 \\
\hline \multirow{5}{*}{ gS0dE0o100 } & 0 & 8.0 & 1.1 & 21.2 & 0.48 & 0.85 & 1 \\
\hline & 180 & 8.0 & 1.1 & 21.1 & 0.47 & 0.87 & 1 \\
\hline & 30 & 7.3 & 1.2 & 20.9 & 0.53 & 0.87 & 38 \\
\hline & 45 & 6.6 & 1.2 & 22.1 & 0.59 & 0.74 & 44 \\
\hline & 60 & 5.8 & 1.2 & 22.9 & 0.65 & 0.56 & 31 \\
\hline
\end{tabular}

\section{Appendix B}

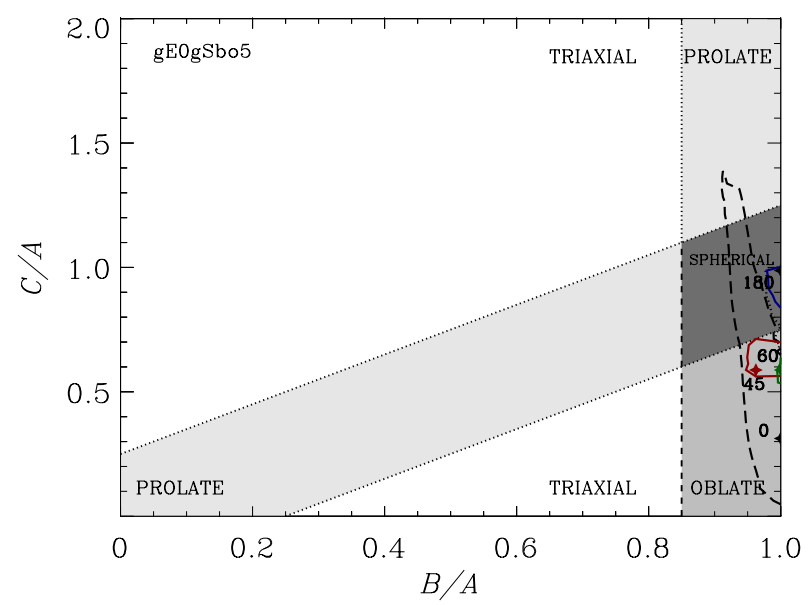

Fig. B.1. As in Fig. 5, but for the remaining simulated lenticular remnants. The most probable $(B / A, C / A)$ values for the simulated lenticular remnant gE0gSbo5 at $\theta=180^{\circ}$ and $\theta=30^{\circ}$ practically overlap. 
A\&A 609, A132 (2018)
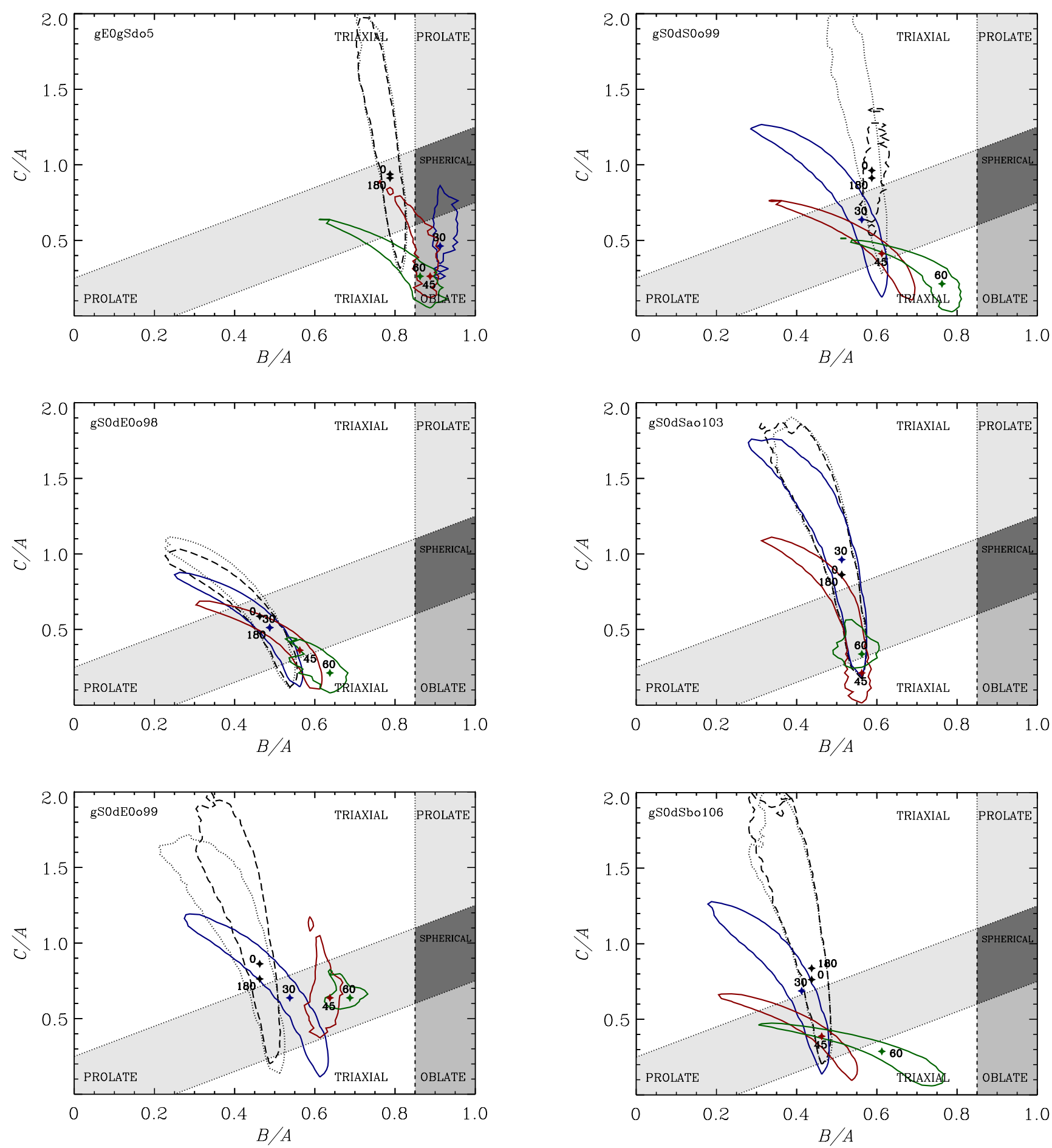

Fig. B.1. continued.

Fig. B.1. continued. 
L. Costantin et al.: The intrinsic shape of bulges in the CALIFA survey
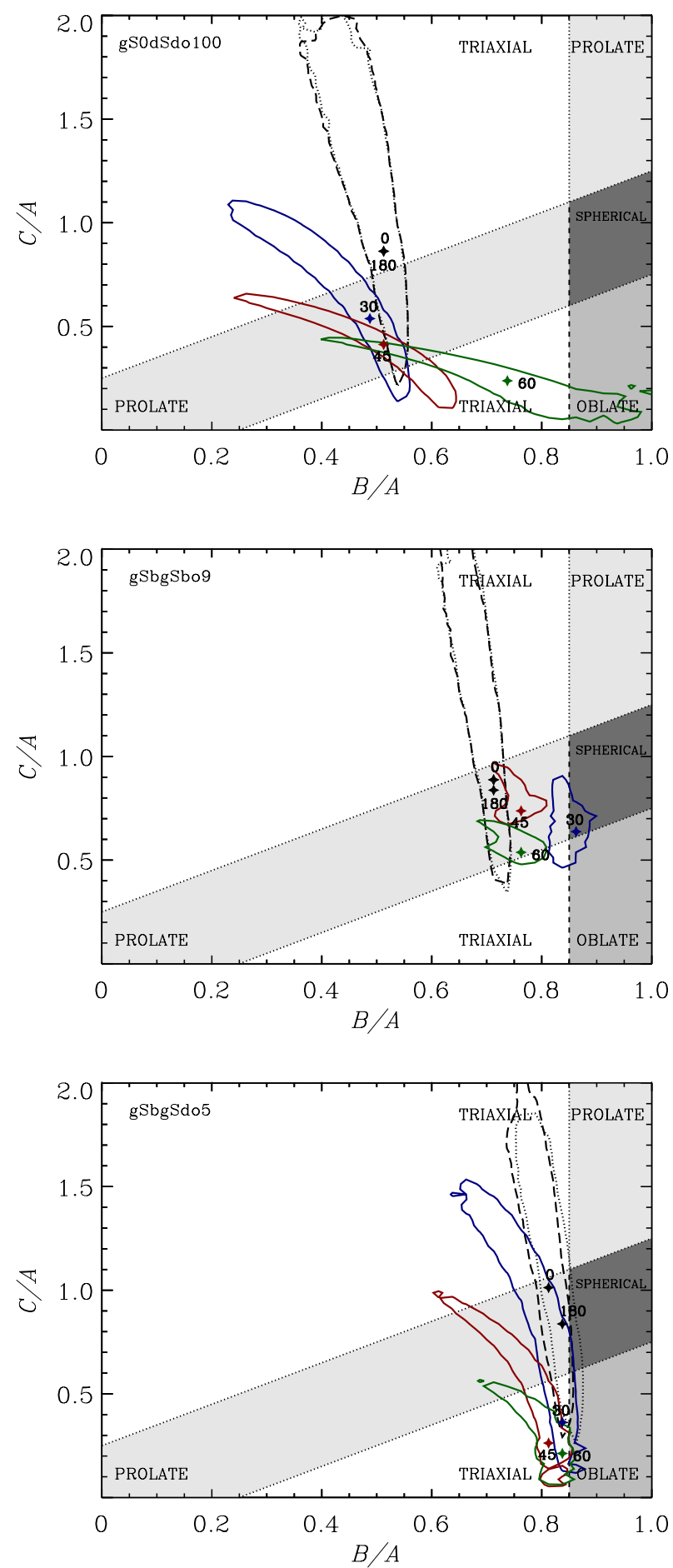

Fig. B.1. continued. 


\section{Appendix C}

Table C.1. Structural parameters of our CALIFA unbarred galaxies.

\begin{tabular}{|c|c|c|c|c|c|c|c|}
\hline $\begin{array}{l}\text { Galaxy } \\
\text { (1) }\end{array}$ & $\begin{array}{c}\log \left(M_{\mathrm{gal}}\right) \\
(2)\end{array}$ & $\begin{array}{c}q_{\mathrm{b}} \\
(3)\end{array}$ & $\begin{array}{c}\mathrm{PA}_{\mathrm{b}} \\
(4)\end{array}$ & $\begin{array}{c}q_{\mathrm{d}} \\
(5)\end{array}$ & $\begin{array}{c}\mathrm{PA}_{\mathrm{d}} \\
(6)\end{array}$ & $\begin{array}{l}B / A \\
(7)\end{array}$ & $\begin{array}{c}C / A \\
(8)\end{array}$ \\
\hline NGC 0001 & 10.6 & $0.79 \pm 0.02$ & $127 \pm 3$ & $0.64 \pm 0.01$ & $94 \pm 1$ & 0.71 & 0.64 \\
\hline NGC 0160 & 10.9 & $0.71 \pm 0.02$ & $50 \pm 4$ & $0.508 \pm 0.003$ & $48.4 \pm 0.2$ & 0.96 & 0.61 \\
\hline NGC 0237 & 10.2 & $0.45 \pm 0.02$ & $47 \pm 3$ & $0.60 \pm 0.01$ & $178 \pm 1$ & 0.29 & 0.41 \\
\hline NGC 0234 & 10.6 & $0.92 \pm 0.02$ & $77 \pm 4$ & $0.861 \pm 0.003$ & $76.8 \pm 0.2$ & 1.00 & 0.71 \\
\hline NGC 0257 & 10.8 & $0.68 \pm 0.01$ & $96 \pm 2$ & $0.621 \pm 0.008$ & $94.1 \pm 0.6$ & 0.96 & 0.44 \\
\hline NGC 0496 & 10.3 & $0.86 \pm 0.05$ & $46 \pm 5$ & $0.57 \pm 0.01$ & $33.4 \pm 0.8$ & 0.91 & 0.81 \\
\hline NGC 0677 & 10.9 & $0.92 \pm 0.01$ & $31 \pm 2$ & $0.820 \pm 0.008$ & $171.2 \pm 0.6$ & 0.91 & 0.91 \\
\hline NGC 0873 & 10.2 & $0.63 \pm 0.01$ & $129 \pm 2$ & $0.840 \pm 0.008$ & $140.2 \pm 0.6$ & 0.66 & 0.54 \\
\hline NGC 1070 & 10.8 & $0.97 \pm 0.02$ & $28 \pm 4$ & $0.814 \pm 0.003$ & $1.5 \pm 0.2$ & 0.96 & 0.94 \\
\hline NGC 1094 & 10.6 & $0.76 \pm 0.03$ & $97 \pm 4$ & $0.688 \pm 0.008$ & $93.1 \pm 0.4$ & 0.96 & 0.49 \\
\hline NGC 1349 & 10.8 & $0.95 \pm 0.02$ & $98 \pm 3$ & $0.88 \pm 0.01$ & $98 \pm 1$ & 1.00 & 0.76 \\
\hline NGC 1665 & 10.5 & $0.80 \pm 0.01$ & $54 \pm 2$ & $0.559 \pm 0.008$ & $48.1 \pm 0.6$ & 0.94 & 0.69 \\
\hline NGC 2476 & 10.5 & $0.71 \pm 0.02$ & $144 \pm 4$ & $0.664 \pm 0.003$ & $148.7 \pm 0.2$ & 0.96 & 0.46 \\
\hline IC 2341 & 10.8 & $0.55 \pm 0.03$ & $5 \pm 4$ & $0.533 \pm 0.008$ & $1.8 \pm 0.4$ & 0.94 & 0.29 \\
\hline NGC 2592 & 10.3 & $0.80 \pm 0.01$ & $58 \pm 2$ & $0.803 \pm 0.008$ & $57.9 \pm 0.6$ & 1.0 & 0.31 \\
\hline NGC 2916 & 10.5 & $0.82 \pm 0.02$ & $7 \pm 4$ & $0.651 \pm 0.003$ & $15.0 \pm 0.2$ & 0.94 & 0.66 \\
\hline NGC 3106 & 11.0 & $0.96 \pm 0.01$ & $144 \pm 2$ & $0.901 \pm 0.008$ & $135.3 \pm 0.6$ & 1.00 & 0.81 \\
\hline NGC 3158 & 11.6 & $0.78 \pm 0.01$ & $70 \pm 2$ & $0.868 \pm 0.008$ & $71.0 \pm 0.6$ & 0.89 & 0.39 \\
\hline UGC 05520 & 9.5 & $0.34 \pm 0.03$ & $112 \pm 4$ & $0.53 \pm 0.02$ & $100 \pm 1$ & 0.46 & 0.24 \\
\hline UGC 07012 & 9.1 & $0.43 \pm 0.05$ & $159 \pm 5$ & $0.58 \pm 0.01$ & $13.8 \pm 0.8$ & 0.34 & 0.29 \\
\hline IC 0776 & 9.3 & $0.64 \pm 0.05$ & $40 \pm 5$ & $0.54 \pm 0.01$ & $91.2 \pm 0.8$ & 0.46 & 0.49 \\
\hline NGC 4711 & 10.3 & $0.66 \pm 0.03$ & $48 \pm 4$ & $0.476 \pm 0.008$ & $41.9 \pm 0.4$ & 0.84 & 0.54 \\
\hline NGC 5376 & & $0.72 \pm 0.02$ & $59 \pm 4$ & $0.577 \pm 0.003$ & $65.3 \pm 0.2$ & 0.91 & 0.56 \\
\hline UGC 09110 & 10.1 & $0.65 \pm 0.02$ & $19 \pm 3$ & $0.44 \pm 0.01$ & $21 \pm 1$ & 0.94 & 0.54 \\
\hline NGC 5732 & 9.9 & $0.72 \pm 0.05$ & $39 \pm 5$ & $0.57 \pm 0.01$ & $40.1 \pm 0.8$ & 1.00 & 0.59 \\
\hline NGC 5772 & 10.8 & $0.80 \pm 0.01$ & $38 \pm 2$ & $0.531 \pm 0.008$ & $36.9 \pm 0.6$ & 1.00 & 0.74 \\
\hline NGC 6060 & 10.8 & $0.51 \pm 0.01$ & $100 \pm 2$ & $0.435 \pm 0.008$ & $100.4 \pm 0.6$ & 1.00 & 0.36 \\
\hline NGC 6155 & 10.1 & $0.45 \pm 0.03$ & $118 \pm 4$ & $0.707 \pm 0.008$ & $146.6 \pm 0.4$ & 0.41 & 0.39 \\
\hline NGC 6301 & 10.8 & $0.63 \pm 0.03$ & $110 \pm 4$ & $0.603 \pm 0.008$ & $109.6 \pm 0.4$ & 1.00 & 0.36 \\
\hline NGC 6314 & 11.1 & $0.51 \pm 0.02$ & $173 \pm 3$ & $0.56 \pm 0.01$ & $175 \pm 1$ & 0.89 & 0.19 \\
\hline NGC 7047 & 10.7 & $0.52 \pm 0.03$ & $111 \pm 4$ & $0.491 \pm 0.008$ & $107.0 \pm 0.4$ & 0.89 & 0.29 \\
\hline UGC 12224 & 9.9 & $0.56 \pm 0.02$ & $103 \pm 3$ & $0.84 \pm 0.01$ & $34 \pm 1$ & 0.51 & 1.04 \\
\hline IC 5309 & 10.2 & $0.41 \pm 0.03$ & $14 \pm 4$ & $0.50 \pm 0.02$ & $26 \pm 1$ & 0.64 & 0.24 \\
\hline NGC 7653 & 10.5 & $0.89 \pm 0.02$ & $20 \pm 3$ & $0.84 \pm 0.01$ & $164 \pm 1$ & 0.89 & 0.81 \\
\hline NGC 7782 & 11.1 & $0.71 \pm 0.01$ & $179 \pm 2$ & $0.556 \pm 0.008$ & $176.5 \pm 0.6$ & 0.96 & 0.56 \\
\hline NGC 5481 & 10.3 & $0.93 \pm 0.02$ & $114 \pm 4$ & $0.738 \pm 0.003$ & $114.8 \pm 0.1$ & 1.00 & 0.86 \\
\hline UGC 09708 & 10.1 & $0.81 \pm 0.05$ & $138 \pm 6$ & $0.76 \pm 0.04$ & $151 \pm 3$ & 0.91 & 0.59 \\
\hline UGC 01370 & 10.6 & $0.55 \pm 0.05$ & $156 \pm 5$ & $0.43 \pm 0.01$ & $156.5 \pm 0.8$ & 0.94 & 0.41 \\
\hline NGC 5145 & 9.9 & $0.58 \pm 0.01$ & $88 \pm 2$ & $0.807 \pm 0.008$ & $56.0 \pm 0.6$ & 0.54 & 0.56 \\
\hline $\mathrm{MCG}-01-52-012$ & 10.3 & $0.47 \pm 0.02$ & $86 \pm 3$ & $0.80 \pm 0.01$ & $43 \pm 1$ & 0.39 & 0.54 \\
\hline UGC 09837 & 9.1 & $0.61 \pm 0.05$ & $31 \pm 5$ & $0.81 \pm 0.01$ & $137.7 \pm 0.8$ & 0.59 & 1.26 \\
\hline NGC 2526 & 10.2 & $0.68 \pm 0.05$ & $142 \pm 5$ & $0.51 \pm 0.01$ & $130.9 \pm 0.8$ & 0.76 & 0.51 \\
\hline MCG +09-22-053 & 9.4 & $0.79 \pm 0.05$ & $93 \pm 5$ & $0.77 \pm 0.01$ & $127.9 \pm 0.8$ & 0.79 & 0.64 \\
\hline
\end{tabular}

Notes. (1) Galaxy name. (2) Stellar mass of the galaxy from Walcher et al. (2014). (3), (4) Apparent axial ratio and position angle of the bulge. (5), (6) Apparent axial ratio and position angle of the disk. (7), (8) Most probable intrinsic axial ratios $B / A$ and $C / A$ of the bulge. 
Table C.2. As in Table C.1, but for barred galaxies.

\begin{tabular}{|c|c|c|c|c|c|c|c|}
\hline $\begin{array}{l}\text { Galaxy } \\
\text { (1) }\end{array}$ & $\begin{array}{c}\log \left(M_{\mathrm{gal}}\right) \\
(2)\end{array}$ & $\begin{array}{c}q_{\mathrm{b}} \\
(3)\end{array}$ & $\begin{array}{c}\mathrm{PA}_{b} \\
(4)\end{array}$ & $\begin{array}{l}q_{\mathrm{d}} \\
(5)\end{array}$ & $\begin{array}{c}\mathrm{PA}_{\mathrm{d}} \\
(6)\end{array}$ & $\begin{array}{l}B / A \\
(7)\end{array}$ & $\begin{array}{c}C / A \\
(8)\end{array}$ \\
\hline NGC 0171 & 10.4 & $0.73 \pm 0.02$ & $124 \pm 3$ & $0.885 \pm 0.004$ & $97.6 \pm 0.3$ & 0.71 & 0.76 \\
\hline NGC 0309 & 10.7 & $0.88 \pm 0.03$ & $136 \pm 3$ & $0.894 \pm 0.007$ & $108.4 \pm 0.3$ & 0.91 & 0.61 \\
\hline NGC 0364 & 10.6 & $0.88 \pm 0.05$ & $45 \pm 6$ & $0.73 \pm 0.01$ & $33.3 \pm 0.7$ & 0.93 & 0.76 \\
\hline NGC 0551 & 10.6 & $0.64 \pm 0.05$ & $126 \pm 6$ & $0.44 \pm 0.01$ & $135.9 \pm 0.7$ & 0.78 & 0.53 \\
\hline NGC 0842 & 10.8 & $0.64 \pm 0.02$ & $137 \pm 3$ & $0.525 \pm 0.004$ & $145.1 \pm 0.3$ & 0.86 & 0.46 \\
\hline NGC 1666 & 10.5 & $0.88 \pm 0.02$ & $138 \pm 3$ & $0.880 \pm 0.004$ & $147.5 \pm 0.3$ & 0.96 & 0.41 \\
\hline NGC 1667 & 10.7 & $0.57 \pm 0.03$ & $171 \pm 3$ & $0.687 \pm 0.007$ & $172.1 \pm 0.3$ & 0.81 & 0.26 \\
\hline UGC 03253 & 10.4 & $0.65 \pm 0.05$ & $92 \pm 6$ & $0.60 \pm 0.01$ & $78.4 \pm 0.7$ & 0.78 & 0.43 \\
\hline NGC 2486 & 10.6 & $0.83 \pm 0.04$ & $85 \pm 5$ & $0.591 \pm 0.009$ & $90.7 \pm 0.5$ & 0.93 & 0.76 \\
\hline UGC 04145 & 10.6 & $0.60 \pm 0.05$ & $135 \pm 6$ & $0.50 \pm 0.01$ & $138.2 \pm 0.7$ & 0.93 & 0.48 \\
\hline NGC 2572 & 10.9 & $0.59 \pm 0.07$ & $126 \pm 13$ & $0.43 \pm 0.02$ & $137.4 \pm 0.9$ & 0.51 & 0.33 \\
\hline NGC 2880 & 10.4 & $0.79 \pm 0.02$ & $129 \pm 3$ & $0.571 \pm 0.003$ & $143.2 \pm 0.1$ & 0.86 & 0.66 \\
\hline NGC 3381 & 9.6 & $0.70 \pm 0.05$ & $80 \pm 6$ & $0.83 \pm 0.01$ & $45.2 \pm 0.7$ & 0.76 & 0.28 \\
\hline NGC 4185 & 10.6 & $0.67 \pm 0.02$ & $173 \pm 3$ & $0.666 \pm 0.004$ & $167.0 \pm 0.3$ & 0.91 & 0.31 \\
\hline NGC 4210 & 10.3 & $0.75 \pm 0.02$ & $78 \pm 3$ & $0.731 \pm 0.004$ & $94.1 \pm 0.3$ & 0.83 & 0.38 \\
\hline NGC 4961 & 9.6 & $0.67 \pm 0.04$ & $111 \pm 5$ & $0.692 \pm 0.009$ & $99.9 \pm 0.5$ & 0.83 & 0.31 \\
\hline NGC 5056 & 10.6 & $0.58 \pm 0.05$ & $97 \pm 6$ & $0.55 \pm 0.01$ & $179.4 \pm 0.7$ & 0.46 & 0.96 \\
\hline NGC 5157 & 11.1 & $0.73 \pm 0.05$ & $114 \pm 6$ & $0.78 \pm 0.01$ & $105.7 \pm 0.7$ & 0.91 & 0.28 \\
\hline NGC 5473 & 10.6 & $0.92 \pm 0.01$ & $137 \pm 2$ & $0.787 \pm 0.003$ & $155.0 \pm 0.1$ & 0.93 & 0.81 \\
\hline IC 0994 & 11.1 & $0.78 \pm 0.05$ & $19 \pm 6$ & $0.51 \pm 0.01$ & $14.6 \pm 0.7$ & 0.91 & 0.71 \\
\hline NGC 5602 & 10.5 & $0.83 \pm 0.08$ & $163 \pm 8$ & $0.52 \pm 0.03$ & $167 \pm 2$ & 1.00 & 0.81 \\
\hline NGC 5720 & 10.8 & $0.82 \pm 0.05$ & $125 \pm 6$ & $0.65 \pm 0.01$ & $129.2 \pm 0.7$ & 1.00 & 0.73 \\
\hline NGC 5735 & 10.1 & $0.78 \pm 0.05$ & $73 \pm 6$ & $0.90 \pm 0.01$ & $32.6 \pm 0.7$ & 0.78 & 0.36 \\
\hline UGC 09492 & 11.1 & $0.65 \pm 0.05$ & $47 \pm 6$ & $0.45 \pm 0.01$ & $54.2 \pm 0.7$ & 0.78 & 0.48 \\
\hline IC 4534 & 10.7 & $0.65 \pm 0.04$ & $158 \pm 5$ & $0.564 \pm 0.009$ & $162.9 \pm 0.5$ & 0.93 & 0.48 \\
\hline NGC 5888 & 11.2 & $0.69 \pm 0.04$ & $154 \pm 5$ & $0.596 \pm 0.009$ & $153.2 \pm 0.5$ & 0.96 & 0.51 \\
\hline UGC 09777 & 10.2 & $0.95 \pm 0.05$ & $129 \pm 5$ & $0.61 \pm 0.02$ & $145.9 \pm 0.9$ & 1.00 & 0.93 \\
\hline NGC 6278 & 10.7 & $0.81 \pm 0.02$ & $123 \pm 3$ & $0.531 \pm 0.004$ & $126.8 \pm 0.3$ & 0.96 & 0.73 \\
\hline NGC 6941 & 10.9 & $0.68 \pm 0.05$ & $118 \pm 6$ & $0.72 \pm 0.01$ & $130.5 \pm 0.7$ & 0.86 & 0.23 \\
\hline UGC 11649 & 10.4 & $0.82 \pm 0.05$ & $132 \pm 6$ & $0.86 \pm 0.01$ & $71.8 \pm 0.7$ & 0.81 & 1.00 \\
\hline NGC 7321 & 10.9 & $0.63 \pm 0.05$ & $15 \pm 6$ & $0.65 \pm 0.01$ & $22.2 \pm 0.7$ & 0.86 & 0.33 \\
\hline UGC 12185 & 10.5 & $0.71 \pm 0.05$ & $141 \pm 5$ & $0.46 \pm 0.02$ & $155.0 \pm 0.9$ & 0.73 & 0.58 \\
\hline NGC 7591 & 10.7 & $0.85 \pm 0.05$ & $167 \pm 6$ & $0.48 \pm 0.01$ & $148.4 \pm 0.7$ & 0.83 & 0.76 \\
\hline NGC 7671 & 10.7 & $0.83 \pm 0.02$ & $142 \pm 3$ & $0.596 \pm 0.004$ & $135.1 \pm 0.3$ & 0.93 & 0.71 \\
\hline NGC 7716 & 10.3 & $0.51 \pm 0.04$ & $56 \pm 5$ & $0.822 \pm 0.008$ & $39.4 \pm 0.4$ & 0.51 & 0.48 \\
\hline UGC 04455 & 10.9 & $0.73 \pm 0.08$ & $174 \pm 8$ & $0.74 \pm 0.03$ & $11 \pm 2$ & 0.81 & 0.41 \\
\hline NGC 6977 & 10.9 & $0.94 \pm 0.05$ & $171 \pm 6$ & $0.83 \pm 0.01$ & $152.7 \pm 0.7$ & 0.96 & 0.88 \\
\hline UGC 12250 & 11.1 & $0.78 \pm 0.04$ & $13 \pm 5$ & $0.626 \pm 0.009$ & $12.7 \pm 0.5$ & 1.00 & 0.63 \\
\hline NGC 5947 & 10.6 & $0.88 \pm 0.04$ & $39 \pm 5$ & $0.811 \pm 0.009$ & $63.7 \pm 0.5$ & 0.88 & 0.71 \\
\hline NGC 2767 & 10.8 & $1.00 \pm 0.04$ & $178 \pm 5$ & $0.733 \pm 0.009$ & $169.6 \pm 0.5$ & 1.0 & 0.98 \\
\hline
\end{tabular}

Notes. (1) Galaxy name. (2) Stellar mass of the galaxy from Walcher et al. (2014). (3), (4) Apparent axial ratio and position angle of the bulge. (5), (6) Apparent axial ratio and position angle of the disk. (7), (8) Most probable intrinsic axial ratios $B / A$ and $C / A$ of the bulge. 